\title{
SOBRE BORDISMO DE APLICAÇÕES HOMOTÓPICAS A IMERSÕES
}

\author{
Isabel Cristina Rossini
}

Orientador: Prof. Dr. Carlos Biasi

Tese apresentada ao Instituto de Ciências Matemáticas de São Carlos USP, como parte dos requisitos para obtenção do título de Doutor em Ciências - Área Matemática - Geometria e Topologia.

$$
\text { USP - São Carlos }
$$

Fevereiro - 1994 


\section{Agradecimentos}

Ao Prof. Dr. Carlos Biasi, pela orientação dedicada e segura desse trabalho, que muito contribuiu para a realização do mesmo.

À Profa. Dra. Alice K. Miwa Libardi, pelo incentivo e pelas valiosas sugestões na elaboração desse trabalho.

Aos Profs. Drs. Daciberg Gonçalves, Dirceu Penteado e Pedro Pergher, pelas discussões proveitosas.

Aos professores do ICMSC-USP, pela acolhida e formação; em particular, Maria Aparecida S. Ruas, Janey Daccach c Ozíride Manzoli Neto.

Aos amigos do Departamento de Matemática do IGCE-UNESP, pelo apoio e estimulo constantes. De modo especial, aos professores Geraldo G. Duarte, João Ivo Bertolo, Solange Mancini, Nativi V. P. Bertolo, Rosa Lúcia S. Baroni e Anízio Perissinotto Jr.

À UNESP, que me proporcionou condições para desenvolver o programa de doutorado.

À CAPES, pelo auxilio financeiro.

À Sarita e à Simone, pelo excelente trabalho de digitação, realizado com muito carinho e atenção. 


\begin{abstract}
In this thesis we determine normal bordism groups of a space $X$ with coefficients in an orientable virtual bundle $\varphi, \Omega_{i}(X: \varphi)$, for $i \leq 3$, in terms of homology groups of $X$ modulo a convenient Serre class of abelian groups.

We also introduce a normal bordism group which can be seen as a group of maps homotopic to an immersion of $m$-dimensional closed manifolds into a fixed connected manifold $N$ of dimension $n$, with $m<n$.

There is an exact sequence involving these groups $f_{m}(N)$ which allows us to determine them up to a group extension, if we consider $N$ a $\pi$-manifold and $3 m<2 n+1$. We present some results about the kernel and the image of the forgetfull homomorphism defined in this exact sequence.
\end{abstract}

We also establish a relationship between regular homotopy and normal bordism. 


\section{Resumo}

Nesse trabalho determinamos grupos de bordismo normal de um espaço $X$ com coeficientes num fibrado virtual orientável $\varphi, \Omega_{i}(X ; \varphi)$, para $i \leq 3$, em termos dos grupos de homologia de $X$ módulo uma conveniente classe de Serre de grupos abelianos.

Introduzimos também um grupo de bordismo normal que pode ser interpretado como um grupo de aplicações homotópicas a imersões de variedades $m$-dimensionais fechadas numa variedade $n$-dimensional fixa $N, \operatorname{com} m<n$.

Existe uma sequência exata envolvendo esses grupos $f_{m}(N)$ que nos possibilita determiná-los a menos de extensão de grupos, quando $N$ é uma $\pi$-variedade e $3 m<2 n+1$. Apresentamos alguns resultados sobre o núcleo e a imagem do homomorfismo de "esquecimento" definido nessa sequência exata.

Estabelecemos também um relacionamento entre homotopia regular e bordismo normal. 


\section{Índice}

Introdução

Capitulo I. Preliminares 1

1. Grupos de bordismo normal com coeficientes num fibrado virtual 2

2. Resultados básicos $\quad 6$

3. Dimensão geométrica 9

4. Uma generalização do Teorema de Whitehead módulo $C$

Capítulo II. Grupos de bordismo normal em dimensões baixas 21

$\begin{array}{ll}\text { 1. Uma sequência exata } & 21\end{array}$

2. Cálculo de grupos de bordismo normal em dimensões baixas 26

3. Aplicações $\quad 30$

4. O caso não orientável 36

Capítulo III. Homotopia regular e bordismo normal 38

1. Relacionamento entre $V_{f}$ e $I[M, N]_{f}$

2. Bordismo de frames 41

3. A função $l_{f} \quad 45$

4. Alguns resultados sobre a imagem de $l_{f} \quad 47$

$\begin{array}{ll}\text { Bibliografia } & 57\end{array}$ 


\section{INTRODUÇÃO}

Nesse trabalho as variedades consideradas são diferenciáveis, de classe $C^{\infty}$, e as aplicações entre elas são contínuas.

Dada uma aplicação entre variedades $f: M^{m} \rightarrow N^{2 m-1}$, com $m>1$, Li Banghe e Peterson provaram que $f$ é sempre homotópica a uma imersão [ 16, T.4.3 ]. Mostraram também que, para cada inteiro positivo $m$, existem variedades fechadas $M^{m}, N^{2 m-2}$ e uma aplicação $f: M^{m} \rightarrow N^{2 m-2}$ que não é homotópica a uma imersão [ 17, cor.2.2 ].

A questão natural que se coloca é: dada uma aplicação $f: M^{m} \rightarrow N^{2 m-i}, i \geq 0$, entre variedades compactas, em que condições a classe de bordismo $[M, f]$ contém uma imersão ? É claro que esse problema é trivial para $i \leq 1$, pois então $f$ é homotópica a uma imersão.

Brown [ 2 ], Liulevicius [ 20 ] e Olk [ 23 ] estudaram esse problema para o caso em que $N$ é um espaço euclidiano. Para o caso geral há resultados recentes obtidos por Szücs [ 27 ] e Gui-Song Li [ 18 ].

Nesse trabalho introduzimos um determinado grupo de bordismo normal que pode ser interpretado como grupo de bordismo de aplicações homótopicas a imersões. $O$ estudo de um homomorfismo de "esquecimento" definido nesse grupo dá informações sobre a questão formulada acima.

No capítulo I apresentamos um estudo pormenorizado sobre grupos de bordismo normal com coeficientes num fibrado virtual ( $\$ \S 1-3$ ), conforme Salomonsen [ 24 ]. Usando esses resultados, obtivemos uma sequência exata envolvendo esses grupos ( II.1.3 ), básica para a análise do homomorfismo de "esquecimento". Além disso, introduzimos no parágrafo 4 os conceitos e resultados sobre classes de Serre de grupos 
abelianos, que serão utilizados no capítulo II para o cálculo de grupos de bordismo normal. Ressaltamos que a generalização do Teorema de Whitehead módulo $\mathcal{C}$, obtida por Biasi e Daciberg ( I.4.7 ), é de fundamental importância nessa computação.

Dados um par de espaços topológicos $(X, A)$, um fibrado virtual $\varphi$ e um inteiro $m \geq 0$, o $m$-ésimo grupo de bordismo normal de $(X, A)$ com coeficientes em $\varphi$ é denotado por $\Omega_{m}(X, A ; \varphi)$. Em geral, cálculos explícitos desses grupos são extremamente difíceis, mesmo em dimensões baixas. Koschorke apresenta em [ 10 ] uma sequência exata que nos possibilita encontrar $\Omega_{m}(X ; \varphi)$ para $m \leq 2$, a menos de extensão de grupos. Em [ 19 ] Gui-Song Li determina $\Omega_{2}(X, A ; \varphi)$, com $A \neq \varnothing$, em termos da homologia de $(X, A)$, a menos de extensão de grupos.

No capitulo Il provamos que $\Omega_{m}(X ; \varphi)$ è $C_{2}$-isomorfo a $H_{m}(X ; \mathrm{Z})$ para $m \leq 2$ e é $C_{2,3}$-isomorfo a $H_{3}(X ; \mathrm{Z})$ para $m=3$, onde $C_{2}$ ( resp. $C_{2,3}$ ) é a classe de Serre de grupos abelianos cujos elementos têm ordem uma potência de 2 ( resp. produtos de potências de 2 e de 3 ), sendo $X$ um complexo $C W$ com somente um número finito de células em cada dimensão e $\varphi$ um fibrado virtual orientável sobre $X$.

Consideremos uma variedade $N$ n-dimensional, conexa e compacta, e o fibrado virtual $\widetilde{\varphi}_{n-m}=\varepsilon^{r+(n-m)}-v_{N}^{r} \times \tilde{\gamma}^{n-m}$ sobre $N \times B S O(n-m)$, onde $v_{N}$ é o fibrado normal estável de $N, \quad \tilde{\gamma}^{n-m}$ é o fibrado universal orientável de posto $n-m$ sobre $B S O(n-m)$ e $\varepsilon^{r+(n-m)}$ é fibrado trivial sobre $N \times B S O(n-m)$. O grupo de bordismo normal $\Omega_{m}\left(N \times B S O(n-m) ; \widetilde{\varphi}_{n-m}\right)$ é interpretado como o grupo de bordismo das aplicações contínuas orientáveis de variedades $m$-dimensionais em $N$ que são homotópicas a imersões. Como aplicação dos resultados obtidos até então, apresentamos uma caracterização para o núcleo do homomorfismo de "esquecimento" $\Omega_{m}\left(N \times B S O(n-m) ; \widetilde{\varphi}_{n-m}\right) \rightarrow \Omega_{m}(N)$ no caso em que $n=2 m-i, i \leq 3, m \equiv i \bmod 2$ e $N$ é uma $\pi$-variedade. Há também uma versão não orientada desses resultados. 
Nosso objetivo no capítulo III é obter um relacionamento entre homotopia regular e bordismo normal. Para uma aplicação contínua $f: M^{m} \rightarrow N^{n}, m<n$, pretendemos comparar o conjunto $I[M, N]_{f}$, das classes de homotopia regular de imersões homotópicas a $f$, com o conjunto $C_{f}$, das classes de bordismo normal de aplicações homotópicas a imersões que são bordantes a $f$. Tal comparação é feita através do conjunto $V_{f}$ das classes de homotopia de frames de codimensão $n-m$ do fibrado normal estável de $f$.

Em [12] , Li Banghe define uma ação em $V_{f}$ determinada pelo grupo fundamental da componente por caminhos de $N^{M}$ que contém $f$, denotado por $\pi_{1}\left(N^{M}, f\right)$. O mesmo autor mostra que, para $n \geq m+2$ e $V_{f} \neq \varnothing$, o conjunto das órbitas dessa ação está em correspondência biunivoca com $I[M, N]_{f}[15]$.

A fim de estabelecer o relacionamento desejado introduzimos o conceito de bordismo de frames de codimensão $n-m$, obtendo novamente 0 grupo $\Omega_{m}\left(N \times B S O(n-m) ; \tilde{\varphi}_{n-m}\right)$. Definimos então uma função $l_{f}: V_{f} \rightarrow \ell_{f}$, a qual se fatora por $\bar{l}_{f}$ ao espaço das órbitas $V_{f} / \pi_{1}\left(N^{M}, f\right)$. Apresentamos exemplos onde essa função não é injetora e também onde não é sobrejetora. Obtivemos também resultados sobre a imagem de $\ell_{f}$. 


\section{CAPÍtulo I}

\section{Preliminares}

Nesse capítulo reunimos os conceitos e resultados essenciais para o desenvolvimento desse trabalho.

Nos parágrafos de 1 a 3 apresentamos um estudo sobre bordismo normal, segundo Salomonsen [ 24 ], que é nossa referência básica para o assunto. A definição de grupo de bordismo normal ( 1.9 ) é introduzida no parágrafo 1 e algumas das propriedades satisfeitas por esses grupos são listadas no parágrafo 2. Para maiores detalhes, além de [ 24 ], indicamos também os trabalhos de Dax [ 5 ] e Koschorke [ 10 ]. No parágrafo 3 temos como principal objetivo a obtenção da sequência exata ( 3.9 ) [ 24, T.6.1 ], básica para o estudo do grupo de bordismo normal de aplicações homotópicas a imersões (II, §3).

Na computação dos grupos de bordismo normal em dimensões baixas ( II, $\S 2$ ), utilizamos a técnica de "esquecer" grupos numa determinada classe. Esse processo é formalizado através de um estudo de grupos "módulo uma classe de Serre" de grupos abelianos. Essas noções são introduzidas no parágrafo 4. Como referência indicamos os textos de $\mathrm{Hu}[8$, cap X ] e de Spanier [ 25, cap 9, §6 ]. O principal resultado apresentado nesse parágrafo é a generalização do Teorema de Whitehead módulo $C$, obtida por Biasi e Daciberg, fundamental para os cálculos.

Nesse trabalho as variedades consideradas são diferenciáveis, de classe $C^{\infty}$, e as aplicações entre elas são continuas. Para uma variedade $M$, denotaremos por $\tau_{M}$ e $v_{M}$ os fibrados tangente e normal estável, respectivamente. Para uma variedade $M$ com bordo, o símbolo $\partial M$ indicará o bordo de $M$. 


\section{Grupos de bordismo normal com coeficientes num fibrado virtual}

1.1 Definição. Seja $X$ um espaço topológico. Um fibrado virtual $\varphi$ sobre $X$ é um par ordenado $\left(\xi_{+}, \xi_{-}\right)$de fibrados vetoriais de base $X$, com postos iguais, e é denotado por $\varphi=\xi_{+}-\xi_{-}$.

Descrevemos a seguir algumas construções envolvendo fibrados virtuais.

1.2 Sejam $\varphi=\xi_{+}-\xi_{-}, \psi=\zeta_{+}-\zeta_{-}$fibrados virtuais sobre $X$.

Os fibrados virtuais $-\varphi$ e $\varphi+\psi$ sobre $X$ são dados por $-\varphi=\xi_{-}-\xi_{+}$e $\varphi+\psi=\xi_{+} \oplus \zeta_{+}-\xi_{-} \oplus \zeta_{-}$.

Se $\psi=\zeta_{+}-\zeta_{-}$é fibrado virtual sobre $Y$, o fibrado virtual $\varphi \times \psi$ sobre $X \times Y$ é dado por

$$
\varphi \times \psi=\xi_{+} \times \zeta_{+}-\xi_{-} \times \zeta_{-} .
$$

Se $f: X \rightarrow Y$ é uma aplicação continua, o fibrado virtual induzido $f^{*} \varphi$ sobre $Y$ é dado por

$$
f^{*} \varphi=f^{*} \xi_{+}-f^{*} \xi_{-} .
$$

Se $f$ é uma inclusão ou uma projeção fibrada, $f^{*} \varphi$ é denotado simplesmente por $\varphi$.

Se $\xi^{k}$ é um fibrado vetorial de posto $k$ sobre $X$, os fibrados virtuais $0_{\xi}$ e $\xi^{0}$ sobre $X$ são dados por $0_{\xi}=\xi^{k}-\xi^{k}$ e $\xi^{0}=\xi^{k}-\varepsilon^{k}$, onde $\varepsilon^{k}$ é o fibrado trivial de posto $k$ sobre $X$. O fibrado virtual $\varepsilon^{k}-\varepsilon^{k}$ é denotado por $0^{k}$.

Seja $(X, A)$ um par de espaços topológicos e seja $\varphi=\xi_{+}^{k}-\xi_{-}^{k}$ um fibrado virtual sobre $X$.

1.3 Definição. Uma $\varphi$-variedade sobre $(X, A)$ de dimensão $m$ é uma terna $m=(M, f, F)$, onde $M$ é uma variedade $m$-dimensional compacta com bordo $\partial M$, 
$f:(M, \partial M) \rightarrow(X, A)$ é uma aplicação de pares e $F: \tau_{M} \oplus f^{*} \xi_{-} \sim \varepsilon^{m} \oplus f^{*} \xi_{+}$é um isomorfismo estável de fibrados. (Isso significa que $F$ é uma classe de equivalência de isomorfismos de fibrados $F^{\prime}: \tau_{M} \oplus f^{*} \xi_{-} \oplus \varepsilon^{N} \cong \varepsilon^{m} \oplus f^{*} \xi_{+} \oplus \varepsilon^{N}$, onde a relação de equivalência é gerada por

(i) $F^{\prime} \sim F^{\prime} \times 1_{\varepsilon^{\prime}}$

(ii) $F^{\prime} \sim F^{\prime \prime}$ se $F^{\prime}$ é homotópico a $F^{\prime \prime}$ através de isomorfismos de fibrados).

1.4 Observação. Para $k \geq 2$ existe uma bijeção entre o conjunto de isomorfismos estáveis de fibrados do tipo $\tau_{M} \oplus f^{*} \xi_{-} \sim \varepsilon^{m} \oplus f^{*} \xi_{+}$e o conjunto de classes de homotopia de isomorfismos.

Seja $M^{m}$ uma variedade com bordo $\partial M$. Usando o Teorema do Colarinho, estabelecemos

1.5 Definição. $O$ bordo da $\varphi$-variedade $m=(M, f, F)$ sobre $(X, A)$ é a $\varphi$ variedade $\partial m=\left(\partial M, f_{\mid \partial M}, \partial F\right)$ sobre $A=(A, \varnothing)$, onde

$$
\partial F: \tau_{\partial M} \oplus f^{*} \xi_{-} \sim \varepsilon^{m-1} \oplus f^{*} \xi_{+}
$$

é um isomorfismo estável de fibrados tal que a composição

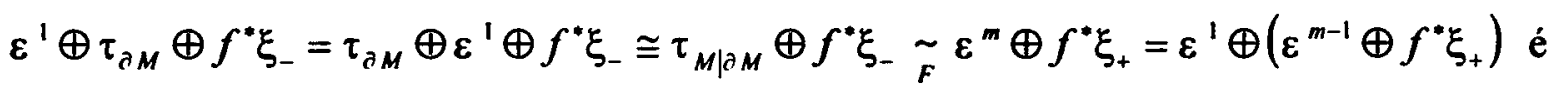
equivalente a $(-1)^{m} \times \partial F$.

Introduzimos a seguir alguns conceitos e construções envolvendo $\varphi$-variedades.

1.6 Sejam $\varphi$, fibrado virtual sobre $X ; g:(Y, B) \rightarrow(X, A)$ aplicação de pares e $m=(M, f, F) \quad g^{*} \varphi$-variedade sobre $(Y, B)$.

A $\varphi$-variedade $g^{*} m$ sobre $(X, A)$ é dada por $g^{*} M=(M, g \circ f, F)$. 
1.7 Sejam $m=(M, f, F), \eta=(N, g, G) \quad \varphi$-variedades sobre $(X, A)$ de mesma dimensão. Então,

$$
\begin{aligned}
& -m=(M, f,-F), \text { onde }-F=F \times(-1): \tau_{M} \oplus f^{*} \xi_{-} \oplus \varepsilon^{\prime} \sim \varepsilon^{m} \oplus f^{*} \xi_{+} \oplus \varepsilon^{\prime} \mathrm{e} \\
& m+\eta=(M \cup N, f \cup g, F \cup G) \text { é dada pela união disjunta. } m+(-\eta) \text { é }
\end{aligned}
$$
denotada por $m-n$.

A variedade vazia define uma $\varphi$-variedade $\theta=(\varnothing, \varnothing, \varnothing)$.

Se $\partial m=-\partial n$, pela identificação dos bordos,

$m \cup \eta=(M \cup N, f \cup g, F \cup G)$ é uma $\varphi$-variedade sobre $(X, \varnothing)$, com $\partial(m \cup n)=\theta$

Vamos introduzir uma relação de equivalência entre $\varphi$-variedades sobre $(X, A)$ como segue:

1.8 Definição. Uma $\varphi$-variedade $m$ sobre $(X, A)$ é bordante a zero, $m \sim Q$, se existe uma $\varphi$-variedade $U$ sobre $(A, A)$ com $\partial U=-\partial m$ e uma $\varphi$-variedade $\mathbb{W}$ sobre $(X, X)$ tais que $\partial W=m \cup U$.

Duas $\varphi$-variedades de mesma dimensão $m, \eta$ sobre $(X, A)$ são bordantes, $m \sim \eta$, se $m-n \sim \theta$.

A classe de equivalência da $\varphi$-variedade $\prod$ sobre $(X, A)$ é denominada classe de bordismo normal de $m$ e é denotada por $[m]$.

1.9 Definição. $O$ conjunto de classes de bordismo de $\varphi$-variedades de dimensão $m$ sobre $(X, A)$, com a estrutura de grupo dada pela união disjunta, é chamado m-ésimo 
grupo de bordismo normal de $(X, A)$ com coeficientes em $\varphi$ e é denotado por $\Omega_{m}(X, A ; \varphi)$.

Mais explicitamente, $\Omega_{m}(X, A ; \varphi)$ tem estrutura de grupo abeliano dada por:

$[m]+[\eta]=[m+\eta] ;-[m]=[-m]$ e $0=[\theta]$.

1.10 Observação. Vamos analisar com mais detalhes a definição ( 1.8 ) no caso em que $A=\varnothing$, que será usado com maior frequência nesse trabalho: duas $\varphi$-variedades $m_{1}=\left(M_{1}, f_{1}, F_{1}\right), m_{2}=\left(M_{2}, f_{2}, F_{2}\right)$ sobre $X$ são bordantes se existe uma $\varphi$-variedade $W=(W, g, G)$ sobre $(X, X)$ tal que $\partial W=m_{1}-m_{2}$, ou seja, $\partial W=M_{1} \cup M_{2}$, $g_{r w}=f_{1} \cup f_{2}$ e $G_{r w}=F_{1} \cup\left(-F_{2}\right)$. Essa última igualdade significa que $\tau_{w \mid a w}$ é identificado com $\tau_{\partial W} \oplus \varepsilon{ }^{\prime}$ através de um campo normal a $\tau_{\partial W}$ que aponta para dentro ao longo de $M_{1}$ e para fora ao longo de $M_{2}$.

Nesse caso, $\Omega_{m}(X ; \varphi)$ é o grupo de bordismo de $m$-variedades singulares em $X$, munidas de uma descrição de seus fibrados tangentes estáveis ( ou normais ) em termos de $\varphi$. Tais grupos também foram estudados por Dax [ 5 ] e Koschorke [ 10 ], que adotam uma convenção de sinal oposta para $\varphi$.

Destacamos a seguir duas funções que são naturalmente definidas entre grupos de bordismo normal e que são homomorfismos.

1.11 Definição. Seja $g:(Y, B) \rightarrow(X, A)$ uma aplicação de pares. $O$ homomorfismo induzido por $\mathrm{g}$

é dado por

$$
g_{*}: \Omega_{m}\left(Y, B ; g^{*} \varphi\right) \rightarrow \Omega_{m}(X, A ; \varphi)
$$

$$
g_{*}([m])=\left[g^{*} M\right] \text {. }
$$




\subsection{Definição. 0 homomorfismo bordo}

$\begin{array}{cc}\partial: \Omega_{m}(X, A ; \varphi) \rightarrow \Omega_{m-1}(A ; \varphi) \\ \text { é dado por } & \partial([m])=[\partial m] .\end{array}$

\section{Resultados Básicos}

Apresentamos a seguir algumas propriedades que são satisfeitas pelos grupos de bordismo normal, definidos no parágrafo 1 , e que serão fundamentais ao desenvolvimento desse trabalho.

Seja $\varphi=\zeta_{+}{ }^{p}-\zeta_{-}{ }^{p}$ um fibrado virtual sobre o espaço $X$.

\subsection{Sequência exata do par $(X, A)$}

Sejam $i: A \rightarrow X, j: X \rightarrow(X, A)$ as inclusões naturais de pares, onde $A=(A, \varnothing)$ e $X=(X, \varnothing)$.

Então, existe uma sequência exata longa

$$
\ldots \rightarrow \Omega_{m}(A ; \varphi) \stackrel{i \cdot}{\rightarrow} \Omega_{m}(X ; \varphi) \stackrel{j .}{\rightarrow} \Omega_{m}(X, A ; \varphi) \stackrel{\partial}{\rightarrow} \Omega_{m-1}(A ; \varphi) \rightarrow \ldots
$$

\subsection{Invariança por homotopia}

Seja $H$ uma homotopia entre as aplicações de pares $f, g:(Y, B) \rightarrow(X, A)$. Então, existe um diagrama comutativo

$$
\begin{aligned}
& \Omega_{m}\left(Y, B ; f^{*} \varphi\right) \stackrel{\theta_{n}}{\cong} \Omega_{m}\left(Y, B ; g^{*} \varphi\right) \\
& \text { f. \ } / \mathrm{g} \text {. } \\
& \Omega_{m}(X, A ; \varphi)
\end{aligned}
$$

onde $\theta_{H}$ é um isomorfismo definido através do isomorfismo $f^{*} \varphi \cong g^{*} \varphi$ determinado por $H$. 
2.3 Seja $\xi$ um fibrado vetorial sobre $X$. Então, existem isomorfismos

e

$$
\begin{aligned}
& \Omega_{m}(X, A ; \varphi) \cong \Omega_{m}\left(X, A ; \varphi+0_{\xi}\right) \\
& \Omega_{m}(X, A ; \varphi) \cong \Omega_{m}\left(X, A ; 0_{\xi}+\varphi\right)
\end{aligned}
$$

dados por

e

$$
\begin{aligned}
& {[M, f, F] \mapsto\left[M, f, F \times 1_{f^{*} \xi}\right]} \\
& {[M, f, F] \mapsto\left[M, f, 1_{f^{*} \xi} \times F\right],}
\end{aligned}
$$

respectivamente.

\subsection{Construção de Pontryagin-Thom}

Se existe um fibrado vetorial $\xi^{k}$ sobre $X$ tal que $\zeta_{+}^{p} \oplus \xi^{k}$ é um fibrado trivial de posto $r=p+k$, então, por $(2.3), \Omega_{m}(X, A ; \varphi) \cong \Omega_{m}\left(X, A ;-\left(\eta^{r}\right)^{0}\right)$, onde $\eta^{r}=\zeta_{-}^{p} \oplus \xi^{k}$.

Nesse caso, a construção de Pontryagin-Thom estabelece um isomorfismo canônico

$$
\Omega_{m}(X, A ; \varphi) \cong \pi_{m+r}^{s}\left(T(\eta), T\left(\eta_{\mid A}\right)\right)
$$

onde $s$ indica homotopia estável e $T(\eta)$ denota o espaço de Thom de $\eta[3, \S 11]$.

Uma demonstração desse resultado é dada por Dax em [ $5, \S 2]$.

2.5 Se $X$ é um complexo $C W$ qualquer, então a inclusão do $(m+1)$-esqueleto $X^{m+1}$ em $X$ induz um isomorfismo entre $\Omega_{m}\left(X^{m+1} ; \varphi \mid X^{m+1}\right)$ e $\Omega_{m}(X ; \varphi)$.

2.6 Seja $X$ um complexo $C W$ com somente um número finito de células em cada dimensão. Então, $\Omega_{m}(X ; \varphi)$ é um grupo abeliano finitamente gerado. 
Demonstração. Pela hipótese, $X^{m+1}$ é compacto; então, existe um fibrado vetorial $\xi^{k}$ sobre $X^{m+1}$ tal que $\zeta_{+}^{p} \oplus \xi^{k}$ é trivial [22, p.71]. Assim, sendo $\eta^{r}=\zeta_{-}^{p} \oplus \xi^{k}$, temos por ( 2.4$)$ e ( 2.5$)$ que

$$
\Omega_{m}(X ; \varphi) \cong \pi_{m+r}^{*}(T(\eta)),
$$

que é um grupo finitamente gerado, visto que $T(\eta)$ è um complexo $C W$ simplesmente conexo finito.

\subsection{Exemplo.}

Seja $X$ um complexo $C W$ conexo por caminhos, com somente um número finito de células em cada dimensão. Então,

$$
\Omega_{0}(X ; \varphi) \cong \begin{cases}Z & , \text { se } w_{1}(\varphi)=0 \\ Z_{2} & , \text { se } w_{1}(\varphi) \neq 0\end{cases}
$$

Demonstração. De forma análoga à demonstração de ( 2.6 ), temos que $\Omega_{0}(X ; \varphi) \cong \pi_{r}^{s}(T(\eta))$, onde $\eta^{r}$ é tal que $\varphi_{\left.\right|^{*}} \cong$ trivial $-\eta^{r}, m \geq 1$. Sendo $T(\eta)$ um espaço $(r-1)$-conexo, $\pi_{r}^{s}(T(\eta)) \cong H_{r}(T(\eta) ; Z)[28, \operatorname{XII}(3.10)]$.

O resultado segue então pelo Teorema do Isomorfismo de Thom-Gysin $[5, \S 5]$.

Nota. Se $\varphi=\zeta_{+}{ }^{p}-\zeta_{-}{ }^{p}$ é um fibrado virtual sobre o espaço $X$, o fibrado orientação de $\varphi$ é dado por $\xi_{\varphi}=\wedge^{p} \zeta_{+}^{p} \otimes \wedge^{p} \zeta_{-}^{p}$, onde $\wedge^{p}$ denota a $p$-ésima potência exterior $[10,6.5]$.

Definimos $w_{1}(\varphi)=w_{1}\left(\zeta_{+}\right)+w_{1}\left(\zeta_{-}\right)$, onde $w_{1}(\eta)$ é a primeira classe de StiefelWhitney do fibrado vetorial $\eta$.

Então, $w_{1}(\varphi)=0$ se, e somente se, o fibrado orientação $\xi_{\varphi}$ é trivial sobre $X$. Nesse caso, dizemos que $\varphi$ é orientável; caso contrário, não orientável. 


\section{Dimensão geométrica}

Seja $\psi=\zeta_{+}{ }^{p}-\zeta_{-}{ }^{p}$ um fibrado virtual sobre um espaço $X$.

3.1 Definição. Uma $k$-redução de $\psi$ é um par $\left(\mu^{k}, G\right)$, onde $\mu^{k}$ é um fibrado vetorial sobre $X \mathrm{e}$

$$
G: \mu^{k} \oplus \zeta_{-} \sim \varepsilon^{k} \oplus \zeta_{+}
$$

é um isomorfismo estável de fibrados.

A dimensão geométrica de $\psi$ é $\leq k$ se, e somente se, $\psi$ possui uma $k$-redução. Denota-se por $\operatorname{gdim} \psi \leq k$.

Observamos que se $\xi^{p}$ é um fibrado vetorial sobre $X$, então o conceito de gdim $\xi$ como fibrado vetorial coincide com $\operatorname{gdim} \xi^{0}$, onde $\xi^{0}=\xi^{p}-\varepsilon^{p}$ é fibrado virtual sobre $X$.

3.2 Exemplo. Sejam $M^{m}, N^{n}$ variedades, $f: M^{m} \rightarrow N^{n}$ uma aplicação contínua, com $m<n$. Em [ 7, T.5.7] Hirsch prova o seguinte

Teorema. $f: M^{m} \rightarrow N^{n}$ é homotópica a uma imersão se, e somente se, existe um fibrado vetorial $\xi^{n-m}$ de base $M$ tal que $f^{*} \tau_{N} \cong \xi^{n-m} \oplus \tau_{M}$. Nesse caso, existe uma imersão $g: M^{m} \rightarrow N^{n}$ tal que $v_{g} \cong \xi^{n-m}$.

Consideremos o fibrado virtual $\psi$ sobre $M$ dado por $\psi=f^{*} \tau_{N}-\varepsilon^{n-m} \oplus \tau_{M}$. Pela definição, temos que $g \operatorname{dim} \psi \leq n-m$ se, e somente se, existe um fibrado vetorial $\mu^{n-m} \quad$ sobre $M$ tal que $\mu^{n-m} \oplus \varepsilon^{n-m} \oplus \tau_{M} \sim \varepsilon^{n-m} \oplus f^{*} \tau_{N}$ é um isomorfismo estável de fibrados. Ou ainda, $\quad \mu^{n-m} \oplus \varepsilon^{n-m} \oplus \tau_{M} \oplus \varepsilon^{\prime} \cong \varepsilon^{n-m} \oplus f^{*} \tau_{N} \oplus \varepsilon^{\prime}$, para algum inteiro $t>0$, o que equivale a $f^{*} \tau_{N} \cong \mu^{n-m} \oplus \tau_{M}$, pois $m<n[9,8(1.5)]$. Pelo Teorema de Hirsch, esse isomorfismo existe se, e somente se, $f$ é homotópica a uma imersão. 
A fim de estudar quando um fibrado virtual $\psi=\zeta_{+}^{p}-\zeta_{-}^{p}$ sobre um espaço $X$ possui $k$-reduções, Salomonsen introduz em $\left[24, \S 6\right.$ ] um fibrado $\tilde{V}_{k}(\Psi) \stackrel{\pi}{\rightarrow} X$ cuja construção descrevemos a seguir.

Vamos construir primeiramente um fibrado $V_{k}(\psi)$ de base $X$ do seguinte modo:

Seja $\Omega=I s o\left(\varepsilon^{k} \oplus \zeta_{-}^{p}, \varepsilon^{k} \oplus \zeta_{+}^{p}\right) \rightarrow X$ o fibrado cuja fibra em $x \in X$ consiste de todos os isomorfismos lineares $\mathbb{R}^{k} \oplus\left(\zeta_{-}\right)_{x} \rightarrow \mathbb{R}^{k} \oplus\left(\zeta_{+}\right)_{x}$. Esse fibrado tem então o grupo $G L(k+p)$ como fibra.

Consideremos a ação à direita do grupo $G L(k)$ em $\Omega$ dada por: se $f \in \Omega$ e $T \in G L(k)$, então $f * T$ é definida na fibra sobre $x \in X$ pela composição

$$
\mathbb{R}^{k} \oplus\left(\zeta_{-}\right)_{x} \stackrel{\mathrm{Txid}_{\left(\zeta_{-}\right)_{x}}}{\longrightarrow} \mathbb{R}^{k} \oplus\left(\zeta_{-}\right)_{x} \stackrel{\boldsymbol{I}_{x}}{\longrightarrow} \mathbb{R}^{k} \oplus\left(\zeta_{+}\right)_{x},
$$

ou seja, $f * T=f \circ\left(T \times 1_{\zeta_{-}}\right) \in \Omega$. Observamos que essa ação é livre.

Definimos então o fibrado $V_{k}(\psi)=\frac{\Omega}{G L(k)} \stackrel{\pi}{\longrightarrow} X$, que tem a variedade de Stiefel $V_{p}\left(\mathbb{R}^{k+p}\right)$ como fibra, a menos de homotopia, que é $(k-1)$-conexa.

Analogamente, para cada inteiro positivo $t$, podemos considerar o fibrado virtual $\psi+0^{\prime}$ e construir o fibrado $V_{k}\left(\psi+0^{\prime}\right) \stackrel{\pi}{\longrightarrow} X$, com fibra $V_{p+c}\left(\mathbb{R}^{k+p+t}\right)$, a menos de homotopia, que também é $(k-1)$-conexa.

Existe uma inclusão $V_{k}(\psi) \rightarrow V_{k}\left(\psi+0^{1}\right)$ dada por $f G L(k) \mapsto\left(f \times 1_{R}\right) G L(k)$, cobrindo a identidade de $X$. Portanto, existe uma sequência de inclusões

$$
V_{k}(\psi) \rightarrow V_{k}\left(\psi+0^{\prime}\right) \rightarrow \ldots \rightarrow V_{k}\left(\psi+0^{\prime}\right) \rightarrow \ldots
$$

cobrindo a identidade de $X$. Assim, fica bem definido o fibrado 
3.3 $\quad \tilde{V}_{k}(\psi)=\bigcup_{i=0} V_{k}\left(\psi+0^{\prime}\right) \stackrel{\pi}{\longrightarrow} X$, com projeção induzida $\pi \quad \mathrm{e}$ $\tilde{V}_{k}=\bigcup_{i=0}^{\infty} V_{p+1}\left(\mathbb{R}^{k+p+1}\right)$ como fibra, também $(k-1)$-conexa.

3.4 Uma vez que $G L(k)$ atua livremente em $\Omega$ e efetivamente em $\mathbb{R}^{k}$, temos que $\frac{\Omega \times \mathbb{R}^{k}}{G L(k)}$ é o espaço total de um fibrado sobre $V_{k}(\psi)=\frac{\Omega}{G L(k)}$, que tem fibra $\mathbb{R}^{k}$.

Esse $k$-fibrado vetorial será denotado por $\mu^{k}$.

Para cada inteiro $t>0$ podemos construir um $k$-fibrado vetorial sobre $V_{k}\left(\psi+0^{t}\right)$ como acima, havendo compatibilidade entre eles. Dessa forma fica induzido um $k$-fibrado vetorial sobre $\tilde{V}_{k}(\psi)$, também denotado por $\mu^{k}$.

3.5 Observação. Existe um isomorfismo canônico $\sigma: \mu^{k} \oplus \pi^{*} \zeta_{-} \rightarrow \varepsilon^{k} \oplus \pi^{*} \zeta_{+}$, onde $\pi$ é a projeção do fibrado $V_{k}(\psi) \rightarrow X$. Basta considerar $\sigma([f, u], v)=f(u, v)$. Isso mostra que $\left(\sigma, \mu^{k}\right)$ é uma $k$-redução de $\pi^{*} \psi$.

Por outro lado, suponhamos que o fibrado $V_{k}(\psi) \rightarrow X$ possua uma seção $s$. Então, a partir de $\sigma, s$ induz o isomorfismo $s^{*} \mu^{k} \oplus \zeta_{-} \cong \varepsilon^{k} \oplus \zeta_{+}$, que fornece uma $k$-redução para $\psi$.

A técnica apresentada acima estabelece uma bijeção entre classes de homotopia de seções do fibrado $V_{k}(\psi) \stackrel{\pi}{\rightarrow} X$ e classes de equivalência $\left(\sigma^{k}, g\right)$, onde $\sigma^{k}$ é um $k$-fibrado vetorial sobre $X$ e $g . \sigma^{k} \oplus \zeta_{-} \cong \varepsilon^{k} \oplus \zeta_{+}$é uma classe de homotopia de isomorfismos. A equivalência é dada por :

$$
\left(\sigma_{1}^{k}, g_{1}\right) \sim\left(\sigma_{2}^{k}, g_{2}\right) \Leftrightarrow \text { existe um isomorfismo } \theta: \sigma_{1} \cong \sigma_{2} \text { tal que } g_{1}=g_{2} \circ\left(\theta \times 1_{\zeta_{-}}\right) \text {. }
$$


3.6 Notação. Seja $\varphi$ um fibrado virtual sobre o espaço $X$. Denotamos por $\Omega_{m}\left(X, \tilde{V}_{k}(\Psi) ; \varphi\right)$ o grupo constituido pelas classes de bordismo normal de ternas $\left(M^{m}, f, F\right)$ que tornam o diagrama

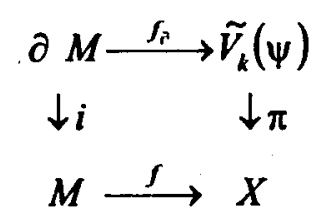

comutativo, ou seja, $f_{\mid \partial M}$ se levanta a $\tilde{V}_{k}(\psi)$ por $f_{\partial}$.

O seguinte resultado é válido:

3.7 Teorema. $O$ homomorfismo induzido pela projeção $\pi: \tilde{V}_{k}(\psi) \rightarrow X$ mergulha na sequência exata longa

$$
\ldots \rightarrow \Omega_{m}\left(\tilde{V}_{k}(\psi) ; \varphi\right) \stackrel{\pi_{m}}{\rightarrow} \Omega_{m}(X ; \varphi) \rightarrow \Omega_{m}\left(X, \tilde{V}_{k}(\psi) ; \varphi\right) \rightarrow \Omega_{m-1}\left(\tilde{V}_{k}(\psi) ; \varphi\right) \rightarrow \ldots
$$

Demonstração. Seja $T_{\pi}$ o cone da projeção $\pi: \tilde{V}_{k}(\psi) \rightarrow X$. Lembramos que existe uma inclusão $i: \tilde{V}_{k}(\psi) \rightarrow T_{\pi}$ e uma projeção $r: T_{\pi} \rightarrow X$ tais que $r \circ i=\pi$. Além disso, $r$ é uma equivalência de homotopia. Temos também que o homomorfismo $r_{*}$, induzido por $r$, estabelece um isomorfismo entre $\Omega_{m}\left(T_{n}, \tilde{V}_{k}(\psi) ; \varphi\right)$ e $\Omega_{m}\left(X, \tilde{V}_{k}(\psi) ; \varphi\right)$, para qualquer fibrado virtual $\varphi$ sobre $X$.

Então, a partir da sequência exata longa de bordismo normal do par $\left(T_{\pi}, \tilde{V}_{k}(\psi)\right)$

$$
\begin{aligned}
& \ldots \rightarrow \Omega_{m}\left(\tilde{V}_{k}(\psi) ; \varphi\right) \stackrel{i^{i} \rightarrow}{\rightarrow} \Omega_{m}\left(T_{\pi} ; \varphi\right) \stackrel{j \cdot}{\rightarrow} \Omega_{m}\left(T_{\pi}, \tilde{V}_{k}(\psi) ; \varphi\right) \stackrel{\text { a }}{\rightarrow} \Omega_{m-1}\left(\tilde{V}_{k}(\psi) ; \varphi\right) \rightarrow \ldots
\end{aligned}
$$

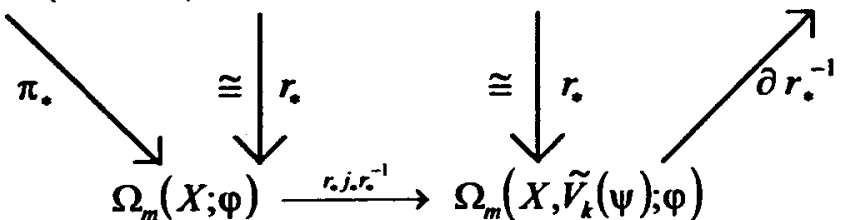

se obtém a sequência exata longa desejada. 
3.8 Observação. Salomonsen define em $[24, \S 7]$ um homomorfismo

$$
\Psi: \Omega_{m}\left(X, \tilde{V}_{k}(\psi) ; \varphi\right) \rightarrow \Omega_{m-k-1}\left(X \times B O(1) ;-k \lambda^{0}-\psi \otimes \lambda+\varphi\right),
$$

onde $\lambda$ é o fibrado de retas canônico sobre o espaço projetivo real $B O(1)$ e $\psi \otimes \lambda=\zeta_{+} \otimes \lambda-\zeta_{-} \otimes \lambda$. Prova também que $\Psi$ é um isomorfismo para $m \leq 2 k+1$ e um epimorfismo para $m=2 k+2[24$, T.7.4 $]$.

A substituição através de $\Psi$ na sequência exata longa obtida no Teorema (3.7), fornece o seguinte

3.9 Teorema. [ 24, T.6.1 ] Existe uma sequência exata

$$
\Omega_{2 k+1}\left(\tilde{V}_{k}(\psi) ; \varphi\right) \stackrel{\pi_{*}}{\rightarrow} \Omega_{2 k+1}(X ; \varphi) \stackrel{\gamma_{k}}{\rightarrow} \Omega_{k}\left(X \times B O(1) ; \Gamma_{k}\right) \stackrel{w_{k}}{\rightarrow} \Omega_{2 k}\left(\tilde{V}_{k}(\psi) ; \varphi\right) \rightarrow \ldots
$$

onde $\Gamma_{k}=-k \lambda^{0}-\psi \otimes \lambda+\varphi$.

\section{Uma generalização do Teorema de Whitehead módulo $C$}

Apresentamos inicialmente os conceitos e resultados necessários para o estudo de grupos módulo uma determinada classe.

4.1 Definição. Uma classe de Serre é uma coleção não vazia $C$ de grupos abelianos que verifica o seguinte axioma: se na sequência exata $A \rightarrow B \rightarrow C$, os grupos $A$, $C$ pertencem a $\mathcal{C}$, então $B$ pertence a $C$.

Equivalentemente, prova-se que 
4.2 Teorema. Uma coleção $C$ de grupos abelianos é uma classe de Serre se, e somente se, satisfaz as seguintes propriedades:

(a) $\mathcal{C}$ contém o grupo trivial.

(b) Se $A \in \mathcal{C}$ e $A^{\prime} \cong A$, então $A^{\prime} \in \mathcal{C}$.

(c) Se $A \subset B$ e $B \in \mathcal{C}$, então $A \in \mathcal{C}$.

(d) Se $A \subset B$ e $B \in C$, então $B / A \in C$.

(e) Se $0 \rightarrow A \rightarrow B \rightarrow C \rightarrow 0$ é uma sequência exata curta, com $A, C \in C$, então $B \in \mathcal{C}$.

Observemos que os ítens (a) e (b) do Teorema ( 4.2 ) mostram que uma classe de Serre não forma um conjunto.

4.3 Listamos a seguir alguns exemplos de classes de Serre:

(1) A classe $A$ de todos os grupos abelianos.

(2) A classe $O$ do grupo trivial.

(3) A classe $A_{f}$ dos grupos abelianos finitamente gerados.

(4) A classe $F$ dos grupos abelianos finitos.

(5) A classe $T$ dos grupos abelianos de torção.

(6) A classe $e_{F}$ dos grupos de torção com componentes $p$-primárias nulas para cada primo $p$ pertencente a uma familia $F$ de números primos dada.

Dada uma classe de Serre $\mathcal{C}$, estamos interessados em calcular módulo grupos em $C$. Introduzimos então a seguinte terminologia:

Um homomorfismo $h: A \rightarrow B$ é chamado um $C$-monomorfismo (resp. $C$ epimorfismo) se ker $h \in \mathcal{C}$ (resp. coker $h \in \mathcal{C}$ ). Se ambas as condições são satisfeitas, $h$ é chamado um $\mathcal{C}$-isomorfismo.

Se existe um $C$-isomorfismo $h: A \rightarrow B$, então $A$ é dito $C$-isomorfo a $B$ e denota-se por $A \stackrel{e}{\cong} B$. 
Observemos que se $C$ é a classe $O$, essas noções coincidem com as correspondentes noções clássicas. Além disso, para uma classe de Serre arbitrária $\mathcal{C}$, essas noções têm as mesmas propriedades formais das noçōes clássicas [ $8, \mathrm{X}$ ( exerc. B ) ].

A fim de provar os teoremas de isomorfismo de Hurewicz e de Whitehead módulo $C$ ( Ver enunciado a seguir), precisamos que as classes de Serre satisfaçam propriedades adicionais. Nesse sentido, introduzimos os seguintes conceitos:

Uma classe de Serre $\mathcal{C}$ é chamada

(1) anel de grupos abelianos: se $A, B \in C$, então $A \otimes B, \operatorname{Tor}(A, B) \in C$.

(2) ideal de grupos abelianos: se $A \in \mathcal{C}$, então $A \otimes B, \operatorname{Tor}(A, B) \in \mathcal{C}$, para $B$ arbitrário.

(3) classe aciclica: se $\pi \in \mathcal{C}$, então $H_{q}(\pi) \in \mathcal{C}$, para $q>0$.

As classes dos exemplos 1, 2, 5 e 6 são ideais de grupos abelianos, enquanto as classes dos exemplos 3 e 4 são anéis de grupos abelianos que não são ideais de grupos abelianos. Todas essas classes são acíclicas.

Usaremos a notação $H_{q}(X)$ para indicar o $q$-ésimo grupo de homologia singular do espaço $X$ com coeficientes inteiros.

4.4 Teorema (do isomorfismo de Hurewicz módulo $C$ absoluto) [ 25; 9, § 6-15].

Seja $\mathcal{C}$ um anel acíclico de grupos abelianos e seja $X$ um espaço simplesmente conexo. As seguintes afirmações são equivalentes:

(i) $\pi_{i}(X) \in \mathcal{C}$ para $2 \leq i<n$.

(ii) $H_{i}(X) \in \mathcal{C}$ para $2 \leq i<n$.

Além disso, qualquer uma delas implica que o homomorfismo de Hurewicz $\mathcal{H}_{i}: \pi_{i}(X) \rightarrow H_{i}(X)$ é um $C$-isomorfismo para $i \leq n$. 
4.5 Teorema (do isomorfismo de Hurewicz módulo $C$ relativo) $[25 ; 9, \S 6-21]$.

Seja $C$ um ideal aciclico de grupos abelianos. Seja $A$ subespaço de $X, \operatorname{com} A$ e $X$ simplesmente conexos. As seguintes afirmações são equivalentes:

(i) $\pi_{i}(X, A) \in \mathcal{C}$ para $2 \leq i<n$.

(ii) $H_{i}(X, A) \in \mathcal{C}$ para $2 \leq i<n$.

Além disso, qualquer uma delas implica que o homomorfismo de Hurewicz $\mathcal{H}_{n}: \pi_{n}(X, A) \rightarrow H_{n}(X, A)$ é um $C$-isomorfismo.

4.6 Teorema ( Whitehead módulo $C$ ) [ 25; 9, §6-22 ].

Seja $e$ um ideal acíclico de grupos abelianos e seja $f: X \rightarrow Y$ uma aplicação entre espaços simplesmente conexos.

Para $n \geq 1$, as seguintes afirmações são equivalentes:

(i) $f_{\#}: \pi_{i}(X) \rightarrow \pi_{i}(Y)$ é um $C$-isomorfismo para $i \leq n$ e é um $C$-epimorfismo para $i=n+1$.

(ii ) $f_{*}: H_{i}(X) \rightarrow H_{i}(Y)$ é um $C$-isomorfismo para $i \leq n$ e é um $\ell$-epimorfismo para $i=n+1$.

O teorema seguinte generaliza o Teorema de Whitehead módulo $C$.

4.7 Teorema. Seja $\mathcal{C}$ um ideal aciclico de grupos abelianos e seja $f: X \rightarrow Y$ uma aplicação continua entre espaços simplesmente conexos. Para $n \geq 1$ as afirmações seguintes são equivalentes:

(i) $f_{\sharp}: \pi_{i}(X) \rightarrow \pi_{i}(Y)$ è $C$ - isomorfismo para $i \leq n$.

(ii) $f_{i}: H_{i}(X) \rightarrow H_{i}(Y)$ é $e$-isomorfismo para $i \leq n$ e o homomorfismo $f_{0} \oplus \mathcal{H}_{n+1}^{Y}: H_{n+1}(X) \oplus \pi_{n+1}(Y) \rightarrow H_{n+1}(Y)$, definido por $f_{*} \oplus \mathcal{H}_{n+1}^{Y}(a, b)=f_{*}(a)+\mathcal{H}_{n+1}^{Y}(b)$, é $\mathcal{C}$ - epimorfismo, onde $\mathcal{H}_{n+1}^{\gamma}$ é homomorfismo de Hurewicz.

Demonstração. A menos de uma equivalência de homotopia, podemos identificar $Y$ com o cilindro da aplicação $f$ e $f$ com a inclusão $i: X \rightarrow Y$. Consideremos 
o diagrama comutativo abaixo, onde as linhas são dadas pelas sequências exatas do par $(Y, X)$ em homotopia e homologia, respectivamente:

$$
\begin{aligned}
& \ldots \rightarrow \pi_{n+1}(X) \stackrel{\left(i_{n+1}\right)_{n}}{\rightarrow} \pi_{n+1}(Y) \stackrel{\left(j_{n+1}\right)_{2}}{\rightarrow} \pi_{n+1}(Y, X) \stackrel{\left(\partial_{n+1}\right)_{n}}{\rightarrow} \pi_{n}(X) \stackrel{\left(i_{n}\right)_{n}}{\rightarrow} \pi_{n}(Y) \rightarrow \ldots \\
& \downarrow \mathcal{H}_{n+1}^{X} \quad \downarrow \mathcal{H}_{n+1}^{Y} \quad \downarrow \mathcal{H}_{n+1} \quad \downarrow \mathcal{H}_{n}^{X} \quad \downarrow \mathcal{H}_{n}^{Y} \\
& \ldots \rightarrow H_{n+1}(X) \stackrel{\left(i_{n+1}\right) \cdot}{\rightarrow} H_{n+1}(Y) \stackrel{\left(j_{n+1}\right) \cdot}{\rightarrow} H_{n+1}(Y, X) \stackrel{\left(\partial_{n+1}\right) \cdot}{\rightarrow} H_{n}(X) \stackrel{\left(i_{n}\right) \cdot}{\rightarrow} H_{n}(Y) \rightarrow \ldots
\end{aligned}
$$

As flechas verticais são homomorfismos de Hurewicz.

$$
\text { Prova de (i) } \Rightarrow \text { (ii) }
$$

Usando a hipótese, segue da sequência de homotopia do par $(Y, X)$ que $\pi_{i}(Y, X) \in$ $\mathcal{C}$ para $i \leq n$. Pelo Teorema de Hurewicz módulo $C$ relativo, segue que $H_{i}(Y, X) \in \mathcal{C}$ para

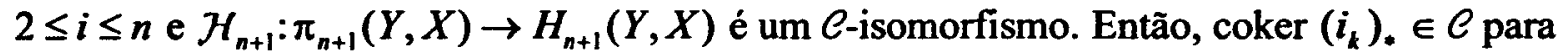
$k \leq n$ e $\operatorname{ker}\left(i_{k}\right) . \in \mathcal{C}$ para $k \leq n-1$.

Mostraremos agora que $\operatorname{Ker}\left(i_{n}\right), \in C$.

Seja $h: \operatorname{coker} \mathcal{H}_{n+1} \rightarrow \frac{\operatorname{ker}\left(i_{n}\right)_{*}}{\mathcal{H}_{n}^{X}\left(\operatorname{ker}\left(i_{n}\right)_{\#}\right)}$ dada por $h(\bar{a})=\overline{\left(\partial_{n+1}\right) .(a)}$. Prova-se facilmente que $h$ está bem definida e é um epimorfismo. Como coker $\mathcal{H}_{\mathrm{n}+1} \in \mathcal{C}$, segue que $\frac{\operatorname{ker}\left(i_{n}\right) .}{\mathcal{H}_{n}^{X}\left(\operatorname{ker}\left(i_{n}\right)_{\|}\right)} \in \mathcal{C}$. Mas, $\operatorname{ker}\left(i_{n}\right)_{\#} \in \mathcal{C} ; \operatorname{logo}, \mathcal{H}_{n}^{x}\left(\operatorname{ker}\left(i_{n}\right)_{\#}\right)$ também pertence a $\mathcal{C}$. Segue então que $\operatorname{ker}\left(i_{n}\right), \in \mathcal{C}$.

Falta provar que $\Psi_{n+1}=\left(i_{n+1}\right) \oplus \mathcal{H}_{n+1}^{Y}: H_{n+1}(X) \oplus \pi_{n+1}(Y) \rightarrow H_{n+1}(Y)$ é um $e$ epimorfismo. Consideremos primeiramente

$$
\overline{\left(j_{n+1}\right)}: \frac{H_{n+1}(Y)}{\operatorname{im} \Psi_{n+1}} \rightarrow \frac{H_{n+1}(Y, X)}{\operatorname{im} \mathcal{H}_{n+1}}
$$

dada por $\overline{\left(j_{n+1}\right)},(\bar{a})=\overline{\left(j_{n+1}\right)(a)}$. Mostra-se facilmente que $\overline{\left(j_{n+1}\right)}$. está bem definido. Então, é exata a sequência 


$$
\operatorname{ker} \overline{\left(j_{n+1}\right)} \stackrel{i}{\longrightarrow} \frac{H_{n+1}(Y)}{\operatorname{im} \psi_{n+1}} \stackrel{\overline{\left(j_{n+1}\right)_{0}}}{\longrightarrow} \frac{H_{n+1}(Y, X)}{\operatorname{im} \mathcal{H}_{n+1}}
$$

Como $C$ é uma classe de Serre e $\mathcal{H}_{n+1}$ é um $C$-epimorfismo, se provarmos que $\operatorname{ker} \overline{\left(j_{n+1}\right) .} \in \mathcal{C}$, então o resultado segue. Para tanto, observemos que $\bar{a} \in \operatorname{ker} \overline{\left(j_{n+1}\right)}$. se, e somente se, existe $b \in \pi_{n+1}(Y, X)$ tal que $\left(j_{n+1}\right),(a)=\mathcal{H}_{n+1}(b)$. Seja, então

$$
k: \operatorname{ker} \overline{\left(j_{n+1}\right)_{*}} \rightarrow \frac{\operatorname{ker}\left(i_{n}\right)_{\#}}{\left(\partial_{n+1}\right)_{\#}\left(\operatorname{ker} \mathcal{H}_{n+1}\right)}
$$

dada por $k(\bar{a})=\overline{\left(\partial_{n+1}\right)_{i t}(b)}$.

( A análise da boa definição de $k$ envolve dois aspectos. Suponhamos que existam $b, b^{\prime} \in \pi_{n+1}(Y, X)$ tais que

$$
\left(j_{n+1}\right)_{*}(a)=\mathcal{H}_{n+1}(b) \text { e }\left(j_{n+1}\right)(a)=\mathcal{H}_{n+1}\left(b^{\prime}\right) .
$$

Então, $b-b^{\prime} \in \operatorname{ker} \mathcal{H}_{n+1} ; \operatorname{logo},\left(\partial_{n+1}\right)_{\#}(b)-\left(\partial_{n+1}\right)_{\|}\left(b^{\prime}\right) \in\left(\partial_{n+1}\right)_{\#}\left(\operatorname{ker} \mathcal{H}_{n+1}\right)$.

Por outro lado, se $\bar{a}=\overline{a^{\prime}}$ e $b, b^{\prime} \in \pi_{n+1}(Y, X)$ são tais que $\left(j_{n+1}\right)_{.}(a)=\mathcal{H}_{n+1}(b)$, $\left(j_{n+1}\right)_{0}\left(a^{\prime}\right)=\mathcal{H}_{n+1}\left(b^{\prime}\right) \quad$ temos $\left(j_{n+1}\right)_{0}(a)-\left(j_{n+1}\right)_{0}\left(a^{\prime}\right)=\left(j_{n+1}\right)_{0} \mathcal{H}_{n+1}^{Y}(c)$, para algum $c \in \pi_{n+1}(Y)$. Então,

$$
\mathcal{H}_{n+1}(b)-\mathcal{H}_{n+1}\left(b^{\prime}\right)=\left(j_{n+1}\right)_{0} \mathcal{H}_{n+1}^{Y}(c)=\mathcal{H}_{n+1}\left(j_{n+1}\right)_{\| 1}(c) .
$$

Isso mostra que $b-b^{\prime}-\left(j_{n+1}\right)_{\#}(c)=c^{\prime}$, com $c^{\prime} \in \operatorname{ker} \mathcal{H}_{n+1}$. Logo,

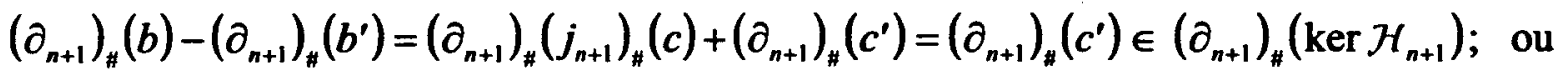
seja, $\left.\overline{\left(\partial_{n+1}\right)_{n}(b)}=\overline{\left(\partial_{n+1}\right)_{n}\left(b^{\prime}\right)}\right)$.

Vamos mostrar que $k$ é um monomorfismo.

Então, $\quad 0 \rightarrow \operatorname{ker} \overline{\left(j_{n+1}\right)} \stackrel{k}{\rightarrow} \frac{\operatorname{ker}\left(i_{n}\right)_{n}}{\left(\partial_{n+1}\right)_{n}\left(\operatorname{ker} \mathcal{H}_{n+1}\right)} \quad$ será $\quad$ exata, onde $\quad 0 \quad \mathrm{e}$ $\frac{\operatorname{ker}\left(i_{n}\right)_{\#}}{\left(\partial_{n+1}\right)_{\#}\left(\operatorname{ker} \mathcal{H}_{n+1}\right)} \in \mathcal{C}$. Consequentemente, $\operatorname{ker} \overline{\left(j_{n+1}\right)_{*}} \in \mathcal{C}$. 
Seja, então, $\bar{a} \in \operatorname{ker} k$. Então, existe $b \in \pi_{n+1}(Y, X)$ tal que $\left(j_{n+1}\right)_{0}(a)=\mathcal{H}_{n+1}(b)$ e $\left(\partial_{n+1}\right)_{\|}(b) \in\left(\partial_{n+1}\right)_{\|}\left(\operatorname{ker} \mathcal{H}_{n+1}\right)$. Assim, $\left(\partial_{n+1}\right)_{\#}(b)=\left(\partial_{n+1}\right)_{n}\left(b^{\prime}\right)$, para algum $\quad b^{\prime} \in \operatorname{ker} \mathcal{H}_{n+1}$. Dessa forma, $b-b^{\prime}=\left(j_{n+1}\right)_{H}(y)$, para algum $y \in \pi_{n+1}(Y)$. Então, $\left(j_{n+1}\right)_{*}(a)=\mathcal{H}_{n+1}(b)=\mathcal{H}_{n+1}\left(b-b^{\prime}\right)=\mathcal{H}_{n+1}\left(j_{n+1}\right)_{n}(y)=\left(j_{n+1}\right)_{*} \mathcal{H}_{n+1}^{\gamma}(y)$. Logo, existe $x \in H_{n+1}(X)$ tal que $a-\mathcal{H}_{n+1}^{\gamma}(y)=\left(i_{n+1}\right) .(x)$, ou seja, $a=\psi_{n+1}(x, y)$.

\section{Prova de ( ii ) $\Rightarrow$ (i)}

Usando a hipótese, segue da sequência de homologia do par $(Y, X)$ que $H_{i}(Y, X) \in$ $C$ para $i \leq n$. Pelo Teorema de Hurewicz módulo $C$ relativo, segue que $\pi_{i}(Y, X) \in C$ para $2 \leq i \leq n$ e $\mathcal{H}_{n+1}: \pi_{n+1}(Y, X) \rightarrow H_{n+1}(Y, X)$ è um $\mathcal{C}$-isomorfismo. Sendo $X$ e $Y$ simplesmente conexos, segue que coker $\left(i_{k}\right)_{*} \in \mathcal{C}$ para $k \leq n$ e $\operatorname{ker}\left(i_{k}\right)_{n} \in \mathcal{C}$ para $k \leq n-1$. Portanto, $\left(i_{k}\right)_{\sharp}: \pi_{k}(X) \rightarrow \pi_{k}(Y)$ é um $\mathcal{C}$-isomorfismo para $k \leq n-1$ e $\left(i_{n}\right)_{\sharp}: \pi_{n}(X) \rightarrow \pi_{n}(Y)$ é um $\mathcal{C}$-epimorfismo.

Falta provar que $\left(i_{n}\right)_{\| t}$ é um $e$-monomorfismo, isto é, que $\operatorname{ker}\left(i_{n}\right)_{\|}=\operatorname{im}\left(\partial_{n+1}\right)_{\#} \cong$ $\frac{\pi_{n+1}(Y, X)}{\operatorname{ker}\left(\partial_{n+1}\right)_{\sharp}}=\frac{\pi_{n+1}(Y, X)}{\operatorname{im}\left(j_{n+1}\right)_{\sharp}} \in C$.

Observamos inicialmente que $\frac{H_{n+1}(Y, X)}{\operatorname{im}\left(j_{n+1}\right)_{*}}=\frac{H_{n+1}(Y, X)}{\operatorname{ker}\left(\partial_{n+1}\right)_{*}} \cong \operatorname{im}\left(\partial_{n+1}\right)_{*}=\operatorname{ker}\left(i_{n}\right)_{*} \in C \mathrm{e}$, portanto, $\left(j_{n+1}\right)_{\text {. }}$ é um $\mathcal{C}$-epimorfismo. Consequentemente, a composta $\left(j_{n+1}\right)_{\bullet} \circ\left(\left(i_{n+1}\right)_{\bullet} \oplus \mathcal{H}_{n+1}^{\gamma}\right)$ é também um $\mathcal{C}$-epimorfismo, usando a hipótese. Mas, $\operatorname{im}\left(j_{n+1}\right)_{\bullet} \circ\left(\left(i_{n+1}\right)_{\odot} \oplus \mathcal{H}_{n+1}^{\gamma}\right) \subset \operatorname{im}\left(j_{n+1}\right)_{\bullet} \circ \mathcal{H}_{n+1}^{Y}$, assim, é exata a sequência

$$
\frac{H_{n+1}(Y, X)}{\operatorname{im}\left(j_{n+1}\right)_{\bullet} \bullet\left(\left(i_{n+1}\right)_{\bullet} \oplus \mathcal{H}_{n+1}^{Y}\right)} \rightarrow \frac{H_{n+1}(Y, X)}{\operatorname{im}\left(j_{n+1}\right)_{\bullet} \circ \mathcal{H}_{n+1}^{Y}} \rightarrow 0,
$$


donde segue que $\left(j_{n+1}\right)_{\circ} \circ \mathcal{H}_{n+1}^{\gamma}$ é um $\mathcal{C}$-epimorfismo. Por outro lado, $\left(j_{n+1}\right) \circ \mathcal{H}_{n+1}^{Y}=\mathcal{H}_{n+1} \circ\left(j_{n+1}\right)_{n}$, onde $\mathcal{H}_{n+1}$ é um $C$-isomorfismo. Segue então que $\left(j_{n+1}\right)_{n}$ é um C-epimorfismo, ou seja, $\frac{\pi_{n+1}(Y, X)}{\operatorname{im}\left(j_{n+1}\right)_{\sharp}} \in \mathcal{C}$. Portanto, $\operatorname{ker}\left(i_{n}\right)_{\sharp} \in \mathcal{C}$.

4.8 Observação. Carlos Biasi e Daciberg Gonçalves provaram o Teorema (4.7) para espaços $C$-nilpotentes, onde $C$ é uma classe generalizada de grupos aciclica ( $\mathrm{A}$ definição e propriedades dessa classe podem ser encontradas em [ 6 ] ). 


\section{CAPÍtulo II}

\section{Grupos de bordismo normal em dimensões baixas}

Nesse capitulo calculamos os grupos de bordismo normal com coeficientes num fibrado virtual orientável, em dimensões $\leq 3$, módulo uma conveniente classe de Serre de grupos abelianos ( T.2.3 ). Os Lemas ( 1.1 ) e ( 2.1 ) são fundamentais nessa computação.

No parágrafo 3 introduzimos um grupo de bordismo normal de aplicações orientáveis homotópicas a imersões, definidas em variedades $m$-dimensionais fechadas numa variedade $n$-dimensional fixa $N$, com $m<n$, que é denotado por $\tilde{d}_{m}(N)$. No caso em que $N$ é uma $\pi$-variedade, esse grupo é determinado a menos de extensão de grupos, quando $3 m<2 n+1 \quad$ ( T.3.1 ). Ainda sob essas hipóteses e como aplicação do cálculo de grupos de bordismo normal, caracterizamos o subgrupo de $\tilde{\delta}_{m}(N)$ constituído pelas aplicações que bordam em $\Omega_{m}(N)$, quando $n=2 m-i, i \leq 3$ e $m \equiv i \bmod 2$ ( T.3.3 ). Há também uma versão não orientada desses resultados ( T.3.8 e T.3.10 ).

O caso não orientável é estudado no parágrafo 4.

Usaremos a notação $H_{q}(X)$ para indicar o $q$-ésimo grupo de homologia singular do espaço $X$ com coeficientes inteiros.

\section{Uma sequência exata}

Usando a teoria desenvolvida por Salomonsen em [ 24 ], em particular os fatos apresentados nos parágrafos 1-3 do capítulo I, obtivemos os resultados que seguem. 
Seja $X$ um complexo $C W$ e seja $\tilde{\varphi}_{r}=\varepsilon^{p+r}-\varepsilon^{p} \times \tilde{\gamma}^{r}$ fibrado virtual sobre $X \times B S O(r)$, onde $\varepsilon^{p}$ é o fibrado trivial de posto $p$ sobre $X$ e $\tilde{\gamma}^{r}$ é o fibrado vetorial orientável universal de posto $r$ sobre $B S O(r)$.

Sob essas hipóteses, temos que

1.1 Lema. Para $r$ suficientemente grande, o $m$-ésimo grupo de bordismo normal $\Omega_{m}\left(X \times B S O(r) ; \tilde{\varphi}_{r}\right)$ é isomorfo ao $m$-ésimo grupo de bordismo orientado de $m$-variedades singulares $\Omega_{m}(X)$, qualquer que seja $m$.

Demonstração. A construção de Pontryagin-Thom ( I.2.4 ) estabelece um isomorfismo entre $\Omega_{m}\left(X \times B S O(r) ; \tilde{\varphi}_{r}\right)$ e $\pi_{m+p+r}^{s}\left(T\left(\varepsilon^{p} \times \tilde{\gamma}^{r}\right)\right)$, onde $T$ denota o espaço de Thom. Por outro lado, $T\left(\varepsilon^{p} \times \tilde{\gamma}^{r}\right)$ é homeomorfo a $T\left(\varepsilon^{p}\right) \wedge M S O(r)$, que por sua vez é homeomorfo a $\sum^{p}(X / \varnothing) \wedge M S O(r)$, onde $M S O(r)=T\left(\tilde{\gamma}^{r}\right), \sum^{p}$ indica a $p$-ésima suspensão e $X / \varnothing$, a uniāo disjunta de $X$ e um ponto. Então,

$$
\Omega_{m}\left(X \times B S O(r) ; \tilde{\varphi}_{r}\right) \cong \pi_{m+p+r}^{s}\left(\sum^{p}(X / \varnothing) \wedge M S O(r)\right)=\pi_{m+r}^{s}(X / \varnothing \wedge M S O(r)) \cong \Omega_{m}(X)
$$

para $r$ suficientemente grande, conforme $[3, \S 12]$.

1.2 Observação. Se $X$ è um complexo $C W$ finito para o qual $H_{*}(X ; Z)$ não tem torção, então o resultado do Lema (1.1) é válido para $\tilde{\varphi}_{r}=\varepsilon^{p+r}-\alpha^{p} \times \tilde{\gamma}^{r}$, onde $\alpha^{p}$ é um fibrado vetorial orientável qualquer sobre $X$. De fato, pela construção de PontryaginThom (I.2.4),

$$
\begin{gathered}
\Omega_{m}\left(X \times B S O(r) ; \tilde{\varphi}_{r}\right) \cong \pi_{m+p+r}^{s}\left(T\left(\alpha^{p} \times \tilde{\gamma}^{r}\right)\right) \cong \\
\pi_{m+p+r}^{s}\left(T\left(\alpha^{p}\right) \wedge M S O(r)\right) \cong \pi_{m+p+r}^{s}\left(T\left(\alpha^{p}\right) / * \wedge M S O(r)\right)
\end{gathered}
$$

onde * é o ponto no infinito do espaço de Thom de $\alpha^{p}$. Por outro lado, para $r$ suficientemente grande,

$$
\pi_{m+p+r}^{s}\left(T\left(\alpha^{p}\right) / * \wedge M S O(r)\right) \cong \Omega_{m+p}\left(T\left(\alpha^{p}\right), *\right) \cong
$$


Temos também que, para $r$ suficientemente grande, $\Omega_{m}\left(X \times B S O(r) ; \tilde{\varphi}_{r}\right) \cong \Omega_{m}(X)$, qualquer que seja $m$, conforme o Lema (1.1).

Por outro lado, a sequência exata ( 1.3.9) para $\tilde{\varphi_{r}}, \tilde{\psi_{r}}$ é dada por

$\Omega_{k}\left(X \times B S O(r) \times B O(1) ; \tilde{\Gamma}_{k}\right) \stackrel{\omega_{2 k}}{\longrightarrow} \Omega_{2 k}\left(\tilde{V}_{k}\left(\tilde{\psi}_{r}\right) ; \tilde{\varphi}_{r}\right) \longrightarrow \ldots$

onde $\tilde{\Gamma}_{k}=-k \lambda^{0}-\tilde{\psi}_{r} \otimes \lambda+\tilde{\varphi}_{r}$.

Portanto, pela substituição nessa sequência de $\Omega_{m}\left(\tilde{V}_{k}\left(\tilde{\psi}_{r}\right) ; \tilde{\varphi}_{r}\right)$ por $\Omega_{m}\left(X \times B S O(k) ; \tilde{\varphi_{k}}\right)$ e $\Omega_{m}\left(X \times B S O(r) ; \tilde{\varphi_{r}}\right)$ por $\Omega_{m}(X)$, através dos isomorfismos acima mencionados, obtemos a sequência exata procurada.

1.4 Corolário. Para $m<2 k+1$, o núcleo do homomorfismo de "esquecimento" $\tilde{F}_{m}$ é $C_{2}$-isomorfo a $\Omega_{m-k}\left(X \times B S O(r) \times B O(1) ; \tilde{\Gamma}_{k}\right)$, onde $e_{2}$ é a classe de Serre dos grupos abelianos cujos elementos têm ordem uma potência de 2 .

Demonstração. Pelo Teorema ( 1.3 ), para $m<2 k+1$, é exata a sequência $\ldots \rightarrow \Omega_{m+1}(X) \stackrel{r_{m+1}}{\longrightarrow} \Omega_{m-k}\left(X \times B S O(r) \times B O(1) ; \tilde{\Gamma}_{k}\right) \stackrel{w_{m}}{\longrightarrow} \Omega_{m}\left(X \times B S O(k) ; \tilde{\varphi}_{k}\right) \stackrel{\tilde{s}_{-}}{\longrightarrow}$
$\Omega_{m}(X) \rightarrow \ldots$

Então, $w_{m}: \Omega_{m-k}\left(X \times B S O(r) \times B O(1) ; \tilde{\Gamma}_{k}\right) \rightarrow \operatorname{im} w_{m}=\operatorname{ker} \tilde{\mathcal{F}}_{m}$ é um epimorfismo cujo núcleo coincide com a imagem de $\gamma_{m+1}$. Conforme [ 24, 10.2 ], os elementos que estão na imagem de $\gamma_{m+1}$ têm ordem uma potência de 2. Portanto, $w_{m}$ é um $C_{2}$-isomorfismo.

Há uma versão não orientada desses resultados se considerarmos o fibrado virtual $\varphi_{r}=\varepsilon^{p+r}-\varepsilon^{p} \times \gamma^{r}$ sobre $X \times B O(r)$, onde $\gamma^{r}$ é o fibrado vetorial universal de posto $r$ sobre $B O(r)$.

Nesse caso, temos 
1.5 Lema. Para $r$ suficientemente grande, o $m$-ésimo grupo de bordismo normal $\Omega_{m}\left(X \times B O(r) ; \varphi_{\mathrm{r}}\right)$ é isomorfo ao $m$-ésimo grupo de $m$-variedades singulares $\eta_{m}(X)$, qualquer que seja $m$.

Demonstração. Segue análoga à do Lema (1.1), observando que $T\left(\gamma^{r}\right)=M O(r)$ e que $\pi_{m+r}^{s}(X / \varnothing \wedge M O(r))$ é isomorfo a $\eta_{m}(X)$, para $r$ suficientemente grande, conforme [ $3, \S 16]$.

1.6 Observação. Se $X$ é um complexo $C W$ finito, então o resultado do Lema (1.5) é válido para $\varphi_{r}=\varepsilon^{p+r}-\alpha^{p} \times \gamma^{r}$, onde $\alpha^{p}$ é um fibrado vetorial qualquer sobre $X$. A demonstração é análoga à da observação (1.2), lembrando que nesse caso $H_{j}\left(X ; \mathrm{Z}_{2}\right) \cong H_{j+p}\left(T\left(\alpha^{p}\right), * ; \mathrm{Z}_{2}\right)$, pelo Teorema do Isomorfismo de Thom, e que $\eta_{m}(X) \cong \sum_{j+k=m} H_{j}\left(X ; Z_{2}\right) \otimes \eta_{k}$, por $[3,17.1]$.

Consideremos os fibrados virtuais $\varphi_{r}=\varepsilon^{p+r}-\varepsilon^{p} \times \gamma^{r}, \psi_{r}=\gamma^{r}-\varepsilon^{r}$ sobre $X \times B O(r)$.

1.7 Teorema. Existe uma sequência exata

$$
\Omega_{2 k+1}\left(X \times B O(k) ; \varphi_{k}\right) \stackrel{J_{k+1}}{\longrightarrow} \eta_{2 k+1}(X) \stackrel{\gamma_{k+1}}{\longrightarrow} \Omega_{m-k}\left(X \times B O(r) \times B O(1) ; \Gamma_{k}\right) \stackrel{w_{2 k}}{\longrightarrow}
$$

$\Omega_{2 k}\left(X \times B O(k) ; \varphi_{k}\right) \rightarrow \ldots$

para $r$ suficientemente grande, onde $\Gamma_{k}=-k \lambda^{0}-\psi_{r} \otimes \lambda+\varphi_{r}$, sendo $\lambda$ o fibrado de retas canônico sobre $B O(1)$.

$\mathcal{F}_{m}$ é um homomorfismo de "esquecimento", $m \leq 2 k+1$. 
Demonstração. Análoga à do Teorema (1.3), usando a sequência (I.3.9) para $\varphi_{r}, \psi_{r}$ e os isomorfismos $\Omega_{m}\left(\tilde{V}_{k}\left(\Psi_{r}\right) ; \varphi_{r}\right) \cong \Omega_{m}\left(X \times B O(k) ; \varphi_{k}\right)$, obtido através de $\theta$, e $\Omega_{m}\left(X \times B O(r) ; \varphi_{r}\right) \cong \eta_{m}(X)$, dado pelo Lema (1.5).

1.8 Corolário. Para $m<2 k+1$, o núcleo do homomorfismo de "esquecimento" $\mathscr{F}_{m}$ é $C_{2}$-isomorfo a $\Omega_{m-k}\left(X \times B O(r) \times B O(1) ; \Gamma_{k}\right)$, onde $C_{2}$ é a classe de Serre dos grupos abelianos cujos elementos têm ordem uma potência de 2 .

Demonstração. Análoga à do Corolário (1.4).

\section{Cálculo de grupos de bordismo normal em dimensões baixas}

Seja $X$ um complexo $C W$ com somente um número finito de células em cada dimensão e seja $\eta^{r}$ um fibrado vetorial estável e orientável sobre $X$, onde $r$ é suficientemente grande.

Consideremos $g: X \rightarrow B S O(r)$ aplicação classificante de $\eta \quad$ e $f: X \rightarrow X \times B S O(r)$ definida por $f=\left(i d_{X}, g\right)$. Seja $\tilde{\varphi}_{r}=\varepsilon^{p+r}-\varepsilon^{p} \times \tilde{\gamma}^{r}$ fibrado virtual sobre $X \times B S O(r)$. Uma vez que $f^{*} \tilde{\varphi}_{r}=-\left(\varepsilon^{p} \oplus \eta^{r}\right)^{0}$, a aplicação $f$ induz um homomorfismo

$$
f_{*}: \Omega_{m}\left(X ;-\left(\varepsilon^{p} \oplus \eta^{r}\right)^{0}\right) \rightarrow \Omega_{m}\left(X \times B S O(r) ; \tilde{\varphi}_{r}\right) \text { para todo } m .
$$

Seja $C_{2}$ (resp. $C_{2,3}$ ) a classe de Serre dos grupos abelianos cujos elementos têm ordens que são potências de 2 (resp. produtos de potências de 2 e de 3 ).

Sob essas hipóteses, temos que 


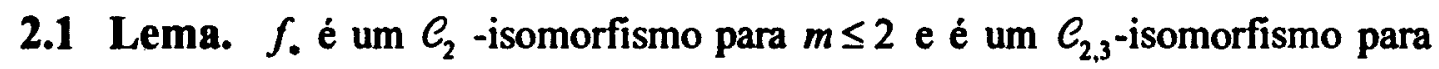
$m=3$.

Demonstração. Sabe-se que $H_{m}(B S O(r)) \in C_{2}$ para $0<m \leq 3$ e $r \geq 4$ [ 1 ]. Então, usando a fórmula de Künneth, podemos concluir que o homomorfismo

$$
f_{.}: H_{m}(X) \rightarrow H_{m}(X \times B S O(r))
$$

é um $C_{2}$ - isomorfismo para $m \leq 3$. Pelo Teorema do Isomorfismo de Thom segue que

$$
T f_{*}: H_{m+p+r}\left(T\left(\varepsilon^{p} \oplus \eta^{r}\right)\right) \rightarrow H_{m+p+r}\left(T\left(\varepsilon^{p} \times \tilde{\gamma}^{r}\right)\right)
$$

é um $C_{2}$-isomorfismo para $m \leq 3$. Em particular, segue que

$$
T f . \oplus \mathcal{H}: H_{3+p+r}\left(T\left(\varepsilon^{p} \oplus \eta^{r}\right)\right) \oplus \pi_{3+p+r}\left(T\left(\varepsilon^{p} \times \tilde{\gamma}^{r}\right)\right) \rightarrow H_{3+p+r}\left(T\left(\varepsilon^{p} \times \tilde{\gamma}^{r}\right)\right)
$$

é um $C_{2}$ - epimorfismo, onde $\not \mathcal{H}$ è homomorfismo de Hurewicz.

Vamos provar agora que o homomorfismo

$$
\text { Tf. } \oplus \mathcal{H}: H_{4+p+r}\left(T\left(\varepsilon^{p} \oplus \eta^{r}\right)\right) \oplus \pi_{4+p+r}\left(T\left(\varepsilon^{p} \times \tilde{\gamma}^{r}\right)\right) \rightarrow H_{4+p+r}\left(T\left(\varepsilon^{p} \times \tilde{\gamma}^{r}\right)\right)
$$

é um $C_{2,3}$ - epimorfismo. É suficiente provar que

$$
\mathcal{H}: \pi_{4+p+r}\left(T\left(\varepsilon^{p} \times \tilde{\gamma}^{r}\right)\right) \rightarrow H_{4+p+r}\left(T\left(\varepsilon^{p} \times \tilde{\gamma}^{r}\right)\right)
$$

é um $C_{2,3}$ - epimorfismo. Existe um diagrama comutativo

$$
\begin{gathered}
\pi_{4+p+r}\left(T\left(\varepsilon^{p} \times \tilde{\gamma}^{r}\right)\right) \stackrel{\#}{\longrightarrow} H_{4+p+r}\left(T\left(\varepsilon^{p} \times \tilde{\gamma}^{r}\right)\right) \\
\phi_{1} \downarrow \\
H_{\phi_{2}}(X) \oplus \pi_{4+r}(M S O(r)) \stackrel{i d \oplus \mathcal{M}}{\longrightarrow} H_{4}(X) \oplus H_{4+r}(M S O(r))
\end{gathered}
$$

onde $\phi_{1}$ é um isomorfismo e $\phi_{2}$ é um $C_{2}$ - isomorfismo obtido pela fórmula de Künneth. Observamos que $H_{4}(X) \oplus \pi_{4+r}(M S O(r))$ é isomorfo a $H_{4}(X) \oplus \Omega_{4} \cong \Omega_{4}(X)$. Então, precisamos apenas mostrar que

$$
\mathcal{H}: \pi_{4+r}(M S O(r)) \rightarrow H_{4+r}(M S O(r))
$$

é um $e_{2,3}$ - epimorfismo.

Consideremos $X$ como um ponto. Nesse caso, o espaço de Thom de $\eta^{r}$ é homeomorfo à esfera $S^{r}$ e o homomorfismo $T g_{\#}: \pi_{m+r}\left(S^{r}\right) \rightarrow \pi_{m+r}(M S O(r))$ é um $C_{2}$ - 
isomorfismo para $m \leq 2$ e é um $e_{2,3}$ - isomorfismo para $m=3$, onde $g$ é aplicação classificante de $\eta$. ( Observamos que $\pi_{3+r}\left(s^{r}\right) \cong Z_{24}$ se $r \geq 5$ ). Pelo Teorema ( 1.4.7) segue que

$$
\operatorname{Tg}_{*}: H_{m+r}\left(S^{r}\right) \rightarrow H_{m+r}(M S O(r))
$$

é um $C_{2}$ - isomorfismo para $m \leq 2$ e é um $C_{2,3}$ - isomorfismo para $m=3$, como também

$$
T g . \oplus \mathcal{H}: H_{4+r}\left(S^{r}\right) \oplus \pi_{4+r}(M S O(r)) \rightarrow H_{4+r}(M S O(r))
$$

é um $C_{2,3}$ - epimorfismo. Sendo $H_{4+r}\left(S^{r}\right)=0$, temos que $T g$. é o homomorfismo nulo e assim,

$$
\mathcal{H}: \pi_{4+r}(M S O(r)) \rightarrow H_{4+r}(M S O(r))
$$

é um $e_{2.3}$ - epimorfismo.

Usando novamente o Teorema ( 1.4.7), podemos concluir que

$$
T f_{\#}: \pi_{m+p+r}\left(\eta\left(\varepsilon^{p} \oplus \eta^{r}\right)\right) \rightarrow \pi_{m+p+r}\left(T\left(\varepsilon^{p} \times \tilde{\gamma}^{r}\right)\right)
$$

é um $C_{2}$ - isomorfismo para $m \leq 2$ e é um $C_{2,3}$ - isomorfismo para $m=3$. Então, pela construção de Pontryagin-Thom ( 1.2.4 ), segue que o homomorfismo induzido por $f$,

$$
f_{*}: \Omega_{m}\left(X ;-\left(\varepsilon^{p} \oplus \eta^{r}\right)^{0}\right) \rightarrow \Omega_{m}\left(X \times B S O(r) ; \tilde{\varphi}_{r}\right)
$$

é um $C_{2}$ - isomorfismo para $m \leq 2$ e é um $C_{2,3}$ - isomorfismo para $m=3$.

2.2 Observação. Uma vez que para $r$ suficientemente grande $\pi_{4+r}\left(S^{r}\right)=0$ e $\pi_{4+r}(M S O(r)) \cong \Omega_{4} \cong Z, \quad$ o homomorfismo $\quad T_{g}: \pi_{4+r}\left(S^{r}\right) \rightarrow \pi_{4+r}(M S O(r)) \quad$ na demonstração do Lema ( 2.1 ) não pode ser um $e_{2,3}$ - epimorfismo. Assim, com as técnicas usadas, o resultado obtido é o melhor possivel.

O resultado principal desse capitulo é dado pelo 
2.3 Teorema. Seja $\varphi$ um fibrado virtual orientável sobre $X$, complexo $C W$ com somente um número finito de células em cada dimensào. Então, o grupo de bordismo normal $\Omega_{m}(X ; \varphi)$ é $C_{2}$-isomorfo a $H_{m}(X)$ para $m \leq 2$ e è $C_{2,3}$-isomorfo a $H_{3}(X)$ para $m=3$.

Demonstração. Por ( 1.2.5) temos que

$$
\Omega_{m}(X ; \varphi) \cong \Omega_{m}\left(X^{m+1} ;\left.\varphi\right|_{X^{m+1}}\right),
$$

onde $X^{m+1}$ denota o $(m+1)$-esqueleto de $X$. Como $X^{m+1}$ é compacto, existe um fibrado vetorial $\eta$ sobre $X^{m+1}$ tal que

$$
\Omega_{m}\left(X^{m+1} ;\left.\varphi\right|^{m+1}\right) \cong \Omega_{m}\left(X^{m+1} ;-\eta^{0}\right) .
$$

Observamos que nesse caso $\eta$ é orientável e pode ser tomado com posto $r$ suficientemente grande. Além disso, por ( I.2.3 ),

$$
\Omega_{m}\left(X^{m+1} ;-\eta^{0}\right) \cong \Omega_{m}\left(X^{m+1} ;-\left(\varepsilon^{p} \oplus \eta^{r}\right)^{0}\right) .
$$

Por outro lado, pelo Lema ( 1.1 ),

$$
\Omega_{m}\left(X^{m+1} \times B S O(r) ; \tilde{\varphi}_{r}\right) \cong \Omega_{m}\left(X^{m+1}\right) .
$$

Sendo $\Omega_{m}\left(X^{m+1}\right) \cong H_{m}\left(X^{m+1}\right)$ para $m \leq 3$ e $H_{m}\left(X^{m+1}\right) \cong H_{m}(X)$, o resultado segue então pelo Lema (2.1).

Nota. A demonstração do Teorema 2.3 mostra que basta ter $X$ complexo $C W$ com um número finito de células em dimensão 4.

2.4 Observação. Para $m=1$, o Teorema (2.3) pode ser obtido por outra técnica, conforme veremos a seguir. Sendo o espaço de Thom de $\eta, T(\eta),(r-1)$-conexo, a seguinte sequência é exata 
$\left(^{*}\right) \quad \pi_{r+2}(T(\eta)) \stackrel{\pi_{2}}{\rightarrow} H_{r+2}(T(\eta)) \stackrel{b}{\rightarrow} \frac{\pi_{r}(T(\eta))}{2 \pi_{r}(T(\eta))} \stackrel{a}{\rightarrow} \pi_{r+1}(T(\eta)) \stackrel{\stackrel{n_{1}}{\rightarrow}}{\rightarrow} H_{r+1}(T(\eta)) \rightarrow 0$ onde $\mathcal{H}_{1}, \mathcal{H}_{2}$ são homomorfismos de Hurewicz [ 28 ]. Para $r$ suficientemente grande, $\pi_{m+r}(T(\eta))$ é isomorfo a $\Omega_{m}\left(X ;-\eta^{0}\right)$, qualquer que seja $m$. Por outro lado, sendo $\eta$ orientável, temos que $\pi_{r}(T(\eta))$ é isomorfo a $\mathrm{Z}$ e $H_{m+r}(T(\eta))$ é isomorfo a $H_{m}(X)$, qualquer que seja $m$. Então, a partir de $\left({ }^{*}\right)$, obtemos a sequência exata

$$
\Omega_{2}\left(X ;-\eta^{0}\right) \rightarrow H_{2}(X) \rightarrow Z_{2} \rightarrow \Omega_{1}\left(X ;-\eta^{0}\right) \rightarrow H_{1}(X) \rightarrow 0,
$$

donde segue que $\Omega_{1}\left(x ;-\eta^{0}\right)$ e $e_{2}$-isomorfo a $H_{1}(x)$.

\section{Aplicações}

Seja $N$ uma variedade $n$-dimensional, conexa e compacta. Consideremos o fibrado virtual $\tilde{\varphi_{k}}=\varepsilon^{p+k}-v_{N}^{p} \times \tilde{\gamma}^{k}$ sobre $N \times B S O(k)$, onde $v_{N}^{p}$ é o fibrado normal estável de $N$.

Denotaremos por $\tilde{e}_{m}(N, k)$ o $m$-ésimo grupo de bordismo normal de $\tilde{\varphi_{k}}$ variedades sobre $N \times B S O(k)$, ou seja, $\tilde{\complement}_{m}(N, k)=\Omega_{m}\left(N \times B S O(k) ; \tilde{\varphi}_{k}\right)$.

Seja $m=(M,(f, g), F)$ representante de um elemento de $\widetilde{C}_{m}(N, k)$. Então, $M \dot{\mathrm{e}}$ uma variedade $m$-dimensional fechada, $f: M \rightarrow N$ e $g: M \rightarrow B S O(k)$ são aplicações contínuas, e $F: \tau_{M} \oplus(f, g)^{*}\left(v_{N}^{p} \times \tilde{\gamma}^{-k}\right) \sim \varepsilon^{m} \oplus(f, g)^{*}\left(\varepsilon^{p+k}\right)$ é um isomorfismo estável de fibrados (Ver I.1.3).

A partir de $F$, sendo $m<n$, temos que

que é equivalente a

$$
\tau_{M} \oplus f^{*} v_{N} \oplus g^{*} \tilde{\gamma}^{k} \sim \varepsilon^{m} \oplus \varepsilon^{p+k}
$$

ou ainda,

$$
\tau_{M} \oplus v_{M} \oplus f^{*}\left(\tau_{N} \oplus v_{N}\right) \oplus g^{*} \tilde{\gamma}^{k} \sim f^{*} \tau_{N} \oplus v_{M} \oplus \varepsilon^{m+p+k}
$$

$$
f^{*} \tau_{N} \oplus v_{M} \sim g^{*} \tilde{\gamma}^{k}
$$


o que mostra que o fibrado normal estável de $f, v_{f}$, admite uma $S O(k)$-estrutura.

No caso $k=n-m, v_{f}$ possuir uma $S O(n-m)$ - estrutura equivale a $f$ ser homotópica a uma imersão, conforme Hirsch [7, T.5.7] (Ver 1.3.2). Além disso, $v_{f}$ é orientável (Nesse caso, dizemos que $f$ é orientável). Assim, podemos interpretar $\tilde{C}_{m}(N, n-m)$ como o grupo de bordismo normal das aplicações continuas orientáveis com contradominio $N$ que são homotópicas a imersões.

Denotaremos $\tilde{\mathcal{C}}_{m}(N, n-m)$ por $\tilde{\rho}_{m}(N)$.

O resultado seguinte é um caso particular do Teorema (1.3) e determina o grupo $\tilde{d}_{m}(N)$ a menos de extensão de grupos.

3.1 Teorema. Seja $N$ uma $\pi$-variedade $n$-dimensional conexa e compacta. Então, para $3 m<2 n+1$, é exata a sequência

$$
\ldots \rightarrow \Omega_{2 m-n}\left(N \times B S O(r) \times B O(1) ; \tilde{\Gamma}_{n-m}\right) \stackrel{w_{m}}{\rightarrow} \tilde{\delta}_{m}(N) \stackrel{\tilde{s}_{m}}{\rightarrow} \Omega_{m}(N) \stackrel{r_{-}}{\rightarrow}
$$

$\Omega_{2 m-n-1}\left(N \times B S O(r) \times B O(1) ; \tilde{\Gamma}_{n-m}\right) \stackrel{n_{m-1}}{\rightarrow} \tilde{\mathcal{C}}_{m-1}(N, n-m) \rightarrow \ldots$

para $r$ suficientemente grande, onde $\tilde{\Gamma}_{n-m}=-(n-m) \lambda^{0}-\tilde{\psi}_{r} \otimes \lambda+\tilde{\varphi}_{r}$, com $\tilde{\varphi}_{r}=\varepsilon^{p+r}-v_{N}^{p} \times \tilde{\gamma}^{r}, \tilde{\psi}_{r}=\tilde{\gamma}^{r}-\varepsilon^{r}$ fibrados virtuais sobre $N \times B S O(r), \lambda$ fibrado de retas canônico sobre $B O(1)$.

Demonstração. É imediata a partir do Teorema (1.3), lembrando que $v_{N}$ é trivial.

Observamos que, nas hipóteses do Teorema (3.1), o núcleo do homomorfismo de "esquecimento" $\widetilde{F}_{m}$ é o grupo das classes de bordismo normal de aplicações contínuas orientáveis com contradomínio $N$ que são homotópicas a imersões e que bordam em $\Omega_{m}(N)$. Nesse caso, temos que 
3.2 Corolário. ker $\tilde{\mathcal{J}}_{m} \cong \Omega_{2 m-n}\left(N \times B S O(r) \times B O(1) ; \tilde{\Gamma}_{n-m}\right)$.

Demonstração. Análoga à do Corolário (1.4).

Usaremos os resultados já obtidos para dar uma caracterização do núcleo de $\widetilde{F}_{m}$, no caso em que $N$ é uma $\pi$-variedade $(2 m-i)$-dimensional, com $i \leq 3$, o qual denotaremos por $\tilde{K}_{m}^{i}$. Nessas condições, temos

3.3 Teorema. Seja $m \equiv i \bmod 2$. Então, $\tilde{K}_{m}^{i}$ é $C_{2}$-isomorfo a $H_{i}(N)$ para $i \leq 2$ e é $e_{2,3}$-isomorfo a $H_{3}(N)$ para $i=3$.

Demonstração. Seja $X=N \times B S O(r) \times B O(1)$. Pelo Corolário (3.2), $\tilde{K}_{m}^{i}$ é $C_{2}$ isomorfo a $\Omega_{i}\left(X ; \tilde{\Gamma}_{m-i}\right)$. Como $X$ é um complexo $C W$ com somente um número finito de células em cada dimensão e $w_{1}\left(\tilde{\Gamma}_{m-i}\right)=(m-i) w_{1}(\lambda)=0$, segue pelo Teorema (2.3) que $\Omega_{i}\left(X ; \tilde{\Gamma}_{m-i}\right)$ é $e_{2}$-isomorfo a $H_{i}(X)$ para $i \leq 2$ e é $e_{2,3}$-isomorfo a $H_{i}(X)$ para $i=3$. Aplicando a fórmula de Künneth a $H_{i}(X)$, obtemos um $C_{2}$ - isomorfismo com $H_{i}(N)$ para $i \leq 3$. Portanto, $\tilde{K}_{m}^{i} \dot{e} C_{2}$-isomorfo a $H_{i}(N)$ para $i \leq 2$ e é $e_{2,3}$ isomorfo a $H_{3}(N)$ para $i=3$.

3.4 Observação. É imediato pelo Teorema (3.3) que $\operatorname{rank} \tilde{K}_{m}^{i}=\boldsymbol{\beta}_{i}(N)$, para $i \leq 3$, onde $\beta_{i}(N)$ é o $i$-ésimo número de Betti inteiro da variedade $N$.

3.5 Observação. No caso do fibrado virtual $\tilde{\Gamma}_{k}=-k \lambda^{0}-\tilde{\psi_{r}} \otimes \lambda+\tilde{\varphi}$, sobre $X=N \times B S O(r) \times B O(1)$, onde $N$ é uma variedade qualquer, $\tilde{\varphi}_{r}=\varepsilon^{p+r}-v_{N}^{p} \times \tilde{\gamma}^{r}$, 
$\tilde{\psi}_{r}=\tilde{\gamma}^{r}-\varepsilon^{r}$ e $\lambda$ é o fibrado de retas canônico sobre $B O(1)$, é possível determinar um fibrado vetorial $\xi$ sobre $X$ de modo que $\tilde{\Gamma}_{+} \oplus \xi$ seja trivial.

De fato, temos que $\tilde{\Gamma}_{+}=\varepsilon^{p+k+r} \oplus r \lambda$ é isomorfo a $\varepsilon^{p+k+r} \oplus \tau_{R P(r-1)} \oplus \varepsilon^{1}$, onde $\operatorname{IRP}(r-1)$ é o espaço projetivo real $(r-1)$-dimensional. Então, $\xi=v_{R P(r-1)}$ satisfaz as condições desejadas.

Nesse caso, sendo $\eta=\tilde{\Gamma}_{-} \oplus \xi=k \lambda \oplus \tilde{\gamma}^{r} \otimes \lambda \oplus v_{N}^{p} \times \tilde{\gamma}^{r} \oplus v_{R P(r-1)}$, temos que $w_{1}\left(\tilde{\Gamma}_{k}\right)=w_{1}(\eta)=k w_{1}(\lambda)+w_{1}(N)$ e $\Omega_{m}\left(X ; \tilde{\Gamma}_{k}\right)$ é isomorfo a $\Omega_{m}\left(X ;-\eta^{0}\right)$, para qualquer $m$.

3.6 Observação. Ainda sob as hipóteses do Teorema (3.1), a imagem do homomorfismo de "esquecimento" $\widetilde{\mathscr{F}}_{m}$ representa o subgrupo de $\Omega_{m}(N)$ constituído pelas classes de bordismo orientado de aplicações contínuas orientáveis $f: M^{m} \rightarrow N^{n}$ que são bordantes a uma imersão.

Nossos resultados permitem determinar essa imagem apenas quando $n=2 m-1$, com $m$ impar. De fato, sendo $X=N \times B S O(r) \times B O(1)$, temos que $\Omega_{0}\left(X ; \tilde{\Gamma}_{m-1}\right) \cong Z$, visto que $\tilde{\Gamma}_{m-1}$ é orientável. Então, pela sequência exata do Teorema (3.1), im $\gamma_{m} \subset Z$ e, como seus elementos têm ordem uma potência de 2 [24, 10.2 ], segue que $\gamma_{m}$ é o homomorfismo nulo. Portanto, im $\tilde{\mathscr{I}}_{m}=\operatorname{ker} \gamma_{m}=\Omega_{m}(N)$, ou seja, toda $f: M^{m} \rightarrow N^{2 m-1}$ orientável é bordante a uma imersão. Todavia, esse fato é uma consequência do resultado obtido por Li Banghe e Peterson em [ 16, T.4.3 ] : toda aplicação contínua $f: M^{m} \rightarrow N^{2 m-1}$ é homotópica a uma imersão, para quaisquer variedades $M, N$.

Em [ 18 ], Gui-Song Li determina condições necessárias e suficientes para que uma aplicação contínua $f: M^{m} \rightarrow N^{2 m-i}$ seja bordante a uma imersão nos casos $i=2,3$.

Destacamos o seguinte resultado no caso $n=2 m-1$, com $m$ impar. 
3.7 Teorema. $\tilde{\delta}_{m}(N) \stackrel{e_{2}}{\cong} H_{1}(N) \oplus \Omega_{m}(N)$.

Demonstração. Pelo Teorema (3.1), obtemos a sequência exata

$$
\ldots \rightarrow \Omega_{1}\left(X ; \tilde{\Gamma}_{m-1}\right) \stackrel{\omega_{m}}{\longrightarrow} \tilde{\tilde{I}}_{m}(N) \stackrel{\tilde{\boldsymbol{s}}_{m}}{\longrightarrow} \Omega_{m}(N) \stackrel{\gamma_{-}}{\longrightarrow} \mathrm{Z} \rightarrow \ldots,
$$

conforme já observamos em (3.5). Então,

$$
\tilde{\jmath}_{m}(N) \cong \tilde{K}_{m}^{1} \oplus \operatorname{im} \tilde{g}_{m}=\tilde{K}_{m}^{1} \oplus \Omega_{m}(N),
$$

também por (3.5). Assim, pelo Teorema (3.3), segue que

$$
\tilde{\rho}_{m}(N) \cong H_{1}(N) \oplus \Omega_{m}(N) \text {. }
$$

Há uma versão não orientada desses resultados se considerarmos inicialmente o fibrado virtual $\varphi_{k}=\varepsilon^{p+k}-v_{N}^{p} \times \gamma^{k}$ sobre $N \times B O(k)$, onde $N$ é uma variedade $n$ dimensional, conexa e compacta, e $\gamma^{k}$ é o fibrado vetorial universal de posto $k$ sobre $B O(k)$.

Denotaremos por $\mathcal{C}_{m}(N, k)$ o $m$-ésimo grupo de bordismo normal de $\varphi_{k}$-variedades sobre $N \times B O(k), \quad \Omega_{m}\left(N \times B O(k) ; \varphi_{k}\right)$. Analogamente ao caso orientado, dado um representante $m=(M,(f, g), F)$ de um elemento desse grupo, se obtém $f^{*} \tau_{N} \oplus v_{M} \sim g^{*} \gamma^{k}$ e, assim, o fibrado normal estável de $f, v_{f}$, admite uma $O(k)$ estrutura. Quando $k=n-m$, $f$ é então homotópica a uma imersão. Assim, $\mathcal{C}_{m}(N, n-m)$ é o grupo de bordismo normal das aplicações contínuas $f: M^{m} \rightarrow N^{n}$ que são homotópicas a imersões. Denotaremos esse grupo por $g_{m}(N)$.

Enunciaremos a seguir a versão não orientada dos resultados já obtidos nesse parágrafo. As demonstrações serão omitidas por serem análogas àquelas apresentadas. Também devemos considerar $N$ uma $\pi$-variedade $n$-dimensional, conexa e compacta. 
3.8 Teorema. Para $3 m<2 n+1$ e $r$ suficientemente grande, é exata a sequência

$$
\ldots \rightarrow \Omega_{2 m-n}\left(N \times B O(r) \times B O(1) ; \Gamma_{n-m}\right) \stackrel{n_{*}}{\rightarrow} d_{m}(N) \stackrel{s_{m}}{\rightarrow} \eta_{m}(N) \stackrel{\gamma_{m}}{\rightarrow}
$$

$\Omega_{2 m-n-1}\left(N \times B O(r) \times B O(1) ; \Gamma_{n-m}\right) \stackrel{w_{m-1}}{\rightarrow} \mathcal{C}_{m-1}(N, n-m) \rightarrow \ldots$

onde $\Gamma_{n-m}=-(n-m) \lambda^{0}-\psi_{r} \otimes \lambda+\varphi_{r}$, com $\varphi_{r}=\varepsilon^{p+r}-v_{N}^{p} \times \gamma^{r}, \psi_{r}=\gamma^{r}-\varepsilon^{r}$ fibrados virtuais sobre $N \times B O(r), \lambda$ fibrado de retas canônico sobre $B O(1)$.

Observamos que nesse caso ker $\mathcal{F}_{m}$ é o grupo de bordismo das aplicações continuas $f: M^{m} \rightarrow N^{n}$ que são homotópicas a imersões e que bordam em $\eta_{m}(N)$.

3.9 Corolário. ker $\mathcal{J}_{m} \stackrel{e_{2}}{\cong} \Omega_{2 m-n}\left(N \times B O(r) \times B O(1) ; \Gamma_{n-m}\right)$.

3.10 Teorema. Seja $n=2 m-i, \operatorname{com} i \leq 3$ e $m \equiv i \bmod 2$. Então, $K_{m}^{i}$ é $e_{2}$ isomorfo a $H_{i}(N)$ para $i \leq 2$ e é $C_{2,3}$-isomorfo a $H_{3}(N)$ para $i=3$.

Observamos apenas que na demonstração desse teorema devemos considerar $X=N \times B O(r) \times B O(1)$. Também podemos concluir que rank $K_{m}^{i}=\beta_{i}(N)$, para $i \leq 3$.

3.11 Observação. A imagem do homomorfismo de "esquecimento" $F_{m}$ representa nesse caso o subgrupo de $\eta_{m}(N)$ constituído pelas classes de bordismo de aplicações contínuas $f: M^{m} \rightarrow N^{n}$ que são bordantes a uma imersão. Também para $n=2 m-1$, com $m$ impar, se obtém im $\mathcal{F}_{m}=\eta_{m}(N)$, ou seja, toda aplicação contínua $f: M^{m} \rightarrow N^{2 m-1}$ é bordante a uma imersão. Esse fato é consequência de [ 16, T.4.3 ].

Além disso, destacamos que

3.12 Teorema. $\delta_{m}(N) \stackrel{\ell_{2}}{\cong} H_{1}(N)$. 
Demonstração. De forma análoga à prova do Teorema (3.7), $d_{m}(N) \stackrel{e_{2}}{\cong} H_{1}(N) \oplus \eta_{m}(N)$. Como $N$ é um complexo $C W$ finito, segue por [ $\left.3,17.1\right]$ que $\eta_{m}(N) \cong \sum_{i=0}^{m} H_{i}\left(N ; Z_{2}\right) \otimes \eta_{m-i}$ e, assim, pertence a $C_{2}$. Portanto, $g_{m}(N) \stackrel{e_{2}}{\cong} H_{1}(N)$.

\section{O caso não orientável}

Seja $X$ um complexo $C W$ com um número finito de células em cada dimensão e seja $\varphi$ um fibrado virtual sobre $X$, não orientável. Nesse caso o resultado do Teorema (2.3) não é válido. Por exemplo, para $X$ conexo por caminhos, temos que $H_{0}(X) \cong Z$, enquanto $\Omega_{0}(X ; \varphi) \cong Z_{2}(1.2 .7)$.

Em dimensão 1 obtivemos a seguinte estimativa:

4.1 Teorema. rank $\Omega_{1}(X ; \varphi)+1 \leq \beta_{1}\left(X, Z_{2}\right)$, onde $\beta_{1}\left(X, Z_{2}\right)$ é o primeiro número de Betti de $X$ com coeficientes $Z_{2}$.

Demonstração. Temos que $\Omega_{1}(X ; \varphi)$ é isomorfo a $\Omega_{1}\left(X^{n} ; \varphi_{\mid X^{n}}\right)$ para $n \geq 2$, onde $X^{n}$ é o $n$-esqueleto de $X$. Sendo $X^{n}$ compacto, $\Omega_{1}\left(X^{n} ; \varphi_{\mid X^{n}}\right) \cong \Omega_{1}\left(X^{n} ;-\eta^{0}\right)$, para algum fibrado vetorial $\eta^{r}$ de posto $r$ sobre $X^{n}$. Observamos que $r$ pode ser tomado suficientemente grande. Além disso, $\Omega_{1}\left(X^{n} ;-\eta^{0}\right)$ é isomorfo a $\pi_{1+r}(T(\eta))$, pela construção de Pontryagin-Thom. Logo, $\Omega_{1}(X ; \varphi) \cong \pi_{1+r}(T(\eta))$.

Uma vez que $\pi_{i}(T(\eta))=0$ para $i \leq r-1$ e $\pi_{r}(T(\eta))$ é isomorfo a $Z_{2}$, temos que $\pi_{i}(T(\eta)) \in C_{2}$ para $1 \leq i \leq r \quad$ e que o homomorfismo de Hurewicz $\mathcal{H}_{r+1}: \pi_{r+1}(T(\eta)) \rightarrow H_{r+1}(T(\eta))$ é um $C_{2}$-isomorfismo. Então, $\Omega_{1}(X ; \varphi)$ é $C_{2}$-isomorfo a $H_{r+1}(T(\eta))$. 
Por outro lado, $H_{1}\left(X ; \mathrm{Z}_{2}\right) \cong H_{1}\left(X^{n} ; \mathrm{Z}_{2}\right) \cong H_{r+1}\left(T(\eta) ; \mathrm{Z}_{2}\right) \cong H_{r+1}(T(\eta)) \otimes \mathrm{Z}_{2} \oplus \mathrm{Z}_{2}$ e, assim,

$$
\beta_{1}\left(X, Z_{2}\right)=\operatorname{dim}_{Z_{2}}\left(H_{r+1}(T(\eta)) \otimes Z_{2}\right)+1 \geq \operatorname{rank} H_{r+1}(T(\eta))+1=\operatorname{rank} \Omega_{1}\left(X ;-\eta^{0}\right)+1
$$

o que conclui a prova.

Como aplicação desse teorema, vamos dar uma estimativa para rank $K_{m}^{1}$ quando $N$ é uma $\pi$-variedade $(2 m-1)$-dimensional, conexa e compacta, com $m$ par. Lembramos que $X=N \times B S O(r) \times B O(1)$ no caso orientado e, na versão não orientada, $X=N \times B O(r) \times B O(1)$, para $r$ suficientemente grande. Temos que:

4.2 Corolário. rank $K_{m}^{1} \leq \beta_{1}\left(N, Z_{2}\right)$ no caso orientado e $\operatorname{rank} K_{m}^{1} \leq \beta_{1}\left(N, Z_{2}\right)+1$ na versão não orientada.

Demonstração. Pelos corolários (3.2) e (3.8), $\operatorname{rank} K_{m}^{1}=\operatorname{rank} \Omega_{1}\left(X ; \Gamma_{m-1}\right)$ nos casos orientado e não orientado, respectivamente. Lembramos que $w_{1}\left(\Gamma_{m-1}\right)=(m-1) w_{1}(\lambda)$, onde $\lambda$ é o fibrado de retas canônico sobre $B O(1)$. Sendo $m$ par, temos que $\Gamma_{m-1}$ não é orientável; então, pelo Teorema (4.1), segue que $\operatorname{rank} K_{m}^{1}+1 \leq \beta_{1}\left(X, Z_{2}\right)$. A conclusão da prova é obtida observando-se que $\beta_{1}\left(X, Z_{2}\right)=\beta_{1}\left(N, Z_{2}\right)+1$ no caso orientado e, $\beta_{1}\left(X, Z_{2}\right)=\beta_{1}\left(N, Z_{2}\right)+2$ na versão não orientada. 


\section{CAPÍtULO III}

\section{Homotopia regular e bordismo normal}

Sejam $\quad M^{m}, N^{n} \quad$ variedades diferenciáveis e $f: M \rightarrow N \quad$ uma aplicação contínua. Vamos supor $M$ fechada, conexa e $\boldsymbol{m}<\boldsymbol{n}$.

Tomemos o fibrado normal estável de $f, v_{f}$, com posto $p$ suficientemente grande. Lembramos que $v_{f}$ é a classe estável de $f^{*} \tau_{N} \oplus v_{M}$, onde $v_{M}$ denota o fibrado normal estável de $M$. Considerando a variedade $M^{m}$ mergulhada em $\mathbb{R}^{m+k}$, com $k \geq m+1$, entendemos " $p$ suficientemente grande" como $p \geq n+m+1$.

Denotaremos por $V_{p-(n-m)}\left(v_{f}\right)$ o fibrado de frames de $v_{f}$ com codimensão igual a $n-m$. Por $V_{f}$ indicaremos o conjunto das classes de homotopia de seções de $V_{p-(n-m)}\left(v_{f}\right)$

Denotaremos por $I[M, N]$, o conjunto das classes de homotopia regular de imersões homotópicas a $f$.

O objetivo desse capitulo é relacionar $I[M, N]_{f}$ com o conjunto das classes de bordismo normal de aplicações homotópicas a imersões que são bordantes a $f$, o qual será denotado por $\mathcal{C}_{f}(\operatorname{Ver} \S 3)$. Isso será feito através de $V_{f}$.

No parágrafo 1 estudamos uma bijeção existente entre $I[M, N]_{f}$ e o espaço das órbitas de $V_{f}$ pela ação de $\pi_{1}\left(N^{M}, f\right)$, grupo fundamental da componente por caminhos de $N^{M}$ que contém $f$. No parágrafo 2 introduzimos o conceito de bordismo de frames de codimensão $n$-m, que nos possibilita definir uma função $\ell_{f}: V_{f} \rightarrow \ell_{f}$. Essa função é estudada nos parágrafos 3 e 4 . 


\section{Relacionamento entre $V_{f}$ e $I[M, N]_{f}$}

Como consequência do Teorema de Hirsch (Ver ( I.3.2 )) temos que

1.1 Teorema. Para $n \geq m+1, V_{f} \neq \varnothing$ se, e somente se, f é homotópica a uma imersão.

Demonstração. Como $n \geq m+1$, existe um fibrado vetorial $\xi^{n-m}$ de base $M$ tal que $f^{*} \tau_{N} \cong \xi^{n-m} \oplus \tau_{M}$ se, e somente se, $f^{*} \tau_{N} \oplus v_{M} \cong \xi^{n-m} \oplus \tau_{M} \oplus v_{M}$, onde $v_{M}$ é o fibrado normal estável de $M$. Isso equivale a $v_{f}^{p} \cong \xi^{n-m} \oplus \varepsilon^{p-(n-m)}$; que por sua vez equivale a $v_{f}$ possuir $p-(n-m)$ seções linearmente independentes. Isso ocorre se, e somente se, $V_{p-(n-m)}\left(v_{f}\right)$ possui uma seção. $O$ resultado segue então pelo Teorema de Hirsch.

Seja $f: M^{m} \rightarrow N^{2 m-i}$ uma aplicação homotópica a imersão. Em [ 14 ], Li Banghe determina $V_{f}$ nos seguintes casos: $i=1, m>3 ; i=2,4<m \equiv 0,1 \bmod 4 ; i=2$, $m=4$ e $w_{1}\left(v_{f}\right)=0$.

Suponhamos $V_{f} \neq \varnothing$.

Denotemos por $N^{M}$ o espaço constituído por todas as aplicações contínuas $M \rightarrow N$ com a topologia compacto-aberta. O grupo fundamental da componente por caminhos de $N^{M}$ que contém $f$ é indicado por $\pi_{1}\left(N^{M}, f\right)$. Tal grupo atua em $V_{f}$ da seguinte maneira:

Seja $[\alpha] \in \pi_{1}\left(N^{M}, f\right)$ e $\alpha: I \rightarrow N^{M}$ um representante de $[\alpha]$, que determina uma homotopia $F: M \times I \rightarrow N$ entre $f$ e $f$; a saber, $F(x, t)=\alpha(t)(x)$. Seja $V_{p-(n-m)}\left(v_{f}^{p}\right)$ o fibrado de frames de $v_{f}^{p}$ com codimensão $n-m$. ( Estamos considerando 
$M \times I$ mergulhada em $\left.\mathbb{R}^{m+k+1}\right)$. Então, $\quad V_{p-(n-m)}\left(v_{F}^{p}\right)_{\mid M \times\{0\}}=V_{p-(n-m)}\left(v_{f}^{p}\right)=$ $=V_{p-(n-m)}\left(v_{F}^{p}\right)_{\mid M \times\{1\}}$.

Seja $\left[s_{o}\right] \in V_{f}$ e $s_{0}$, seção de $V_{p-(n-m)}\left(v_{f}\right)$, representante de $\left[s_{o}\right]$. Pelas considerações acima, o diagrama

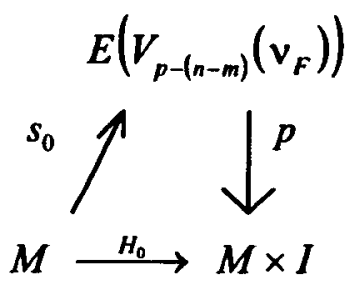

é comutativo, onde $H_{0}(x)=(x, 0)$. Isso mostra que a homotopia $H: M \times I \rightarrow M \times I$, $H(x, t)=(x, t)$, se levanta no nível 0 . Pela propriedade de levantamento de homotopia do fibrado $V_{p-(n-m)}\left(v_{F}\right)$, existe uma homotopia $s: M \times I \rightarrow E\left(V_{p-(n-m)}\left(v_{F}\right)\right)$ tal que $p \circ s=H$ e $s(x, 0)=s_{0}(x)$. (Observamos que, sendo $H=i d_{M \times l}, s$ é efetivamente uma seção de $\left.V_{p-(n-m)}\left(v_{F}\right)\right)$.

Então, para cada $t \in I, s_{t}$ é uma seção de $V_{p-(n-m)}\left(v_{F_{t}}\right)$, onde $F_{t}=F_{\mid M \times\{t\}}$. Em particular, $s_{1}$ é uma seção de $V_{p-(n-m)}\left(v_{f}\right)$. Assim, tem sentido a seguinte

1.2 Definição. A classe de homotopia $\left[s_{1}\right] \in V_{f}$ é a imagem de $[\alpha] \in \pi_{1}\left(N^{M}, f\right)$ atuando em $\left[s_{0}\right] \in V_{r}$.

A órbita de $[s] \in V_{f}$ pela ação de $\pi_{1}\left(N^{M}, f\right)$ será denotada por $\{[s]\}$.

1.3 Observação. Li Banghe prova em [ 15 ] que para $n>m+2$ existe uma bijeção entre o conjunto $V_{f} / \pi_{1}\left(N^{M}, f\right)$ das órbitas dessa ação e o conjunto $I[M, N]_{f}$ das classes de homotopia regular de imersões homotópicas a f. 
Quando essa ação é trivial, fica então estabelecida uma bijeção entre $V_{f}$ e $I[M, N]_{f}$. Isso acontece, por exemplo, se $N$ é um espaço projetivo real de dimensão impar, ou mais geral, se $N$ é um espaço de "lens" $S_{m}^{n}=S^{n} / \mathrm{Z}_{m}, \operatorname{com} n$ ímpar, $n>m+1$ [ 13, T.1 ]. Esse também é o caso quando o fibrado tangente a $N, \tau_{N}$, é estavelmente trivial sobre o $(m+1)$-esqueleto de $N[12$, T.5].

\section{Bordismo de frames}

Seja $N$ uma variedade $n$-dimensional conexa e compacta.

Vamos considerar ternas $(M, g, s)$, onde $M$ é uma variedade $m$-dimensional fechada, com $m<n ; g: M \rightarrow N$ é uma aplicação orientável (i.é , $w_{1}\left(v_{g}^{p}\right)=0$ ) e $s: M \rightarrow E\left(V_{p-(n-m)}\left(v_{g}^{p}\right)\right)$ é uma seção. Para que o conjunto de tais ternas seja não vazio, as aplicações em questão devem ser homotópicas a imersões ( 1.1 ).

Considerando as variedades de dimensão $m$ mergulhadas em $\pi R^{m+k}$ e as de dimensão $m+1$ em $\mathbb{R}^{m+k+1}$, com $k$ suficientemente grande, estabelecemos uma relação de equivalência no conjunto das ternas $(M, g, s)$ como acima da seguinte maneira:

2.1 Definição. Um bordismo entre $\left(M_{1}^{m}, g_{1}, s_{1}\right)$ e $\left(M_{2}^{m}, g_{2}, s_{2}\right)$ é uma terna $\left(W^{m+1}, g, s\right)$ na qual $\partial W=M_{1} \cup M_{2}, \quad g: W \rightarrow N$ é uma aplicação tal que $g_{\mid \partial W}=g_{1} \cup g_{2}$ e $s: W \rightarrow E\left(V_{p-(n-m)}\left(v_{g}^{p}\right)\right)$ é uma seção tal que $s_{\mid \partial W}=s_{1} \cup\left(-s_{2}\right)$, onde $\left(-s_{2}\right)$ denota a troca de sinal em uma das componentes de $s_{2}$ previamente fixada.

Essa definição fornece uma interpretação, em termos de frames de codimensão $n-m$, do grupo de bordismo normal $\tilde{f}_{m}(N)=\Omega_{m}\left(N \times B S O(n-m) ; \tilde{\varphi}_{n-m}\right)$, onde 
$\tilde{\varphi}_{n-m}=\varepsilon^{l+(n-m)}-v_{N}^{\prime} \times \tilde{\gamma}^{n-m}$ é fibrado virtual sobre $N \times B S O(n-m)$ (Ver II.3).

Formalmente temos:

2.2 Proposição. Existe uma bijeção entre o conjunto das classes de bordismo determinadas pela definição ( 2.1 ) e o conjunto $\tilde{g}_{m}(N)$.

Demonstração. Seja $[M, g, s]$ uma classe de equivalência determinada pela definição ( 2.1 ). A seção $s$ de $V_{p-(n-m)}\left(v_{g}^{p}\right)$ fornece $s_{1}, \ldots, s_{p-(n-m)}$ seções linearmente independentes de $v_{g}^{p}$. Tais seções determinam uma decomposição do fibrado normal estável de $g$, a saber: $v_{g}^{p} \cong \xi_{s}^{n-m} \oplus \varepsilon_{s}^{p-(n-m)}$, onde para cada $x \in M$, a fibra de $\varepsilon_{s}^{p-(n-m)}$ é o subespaço gerado por $s_{1}(x), \ldots, s_{p-(n-m)}(x)$ e a de $\xi_{s}^{n-m}$ é o complemento ortogonal desse subespaço em $\left(v_{g}\right)_{x}$. Observamos que, sendo $v_{g}$ um fibrado orientável, $\xi_{s}^{n-m}$ também o é.

Tomemos $h: M \rightarrow B S O(n-m)$ aplicação classificante de $\xi_{s}^{n-m}$. Obtemos então

$$
g^{*} \tau_{N} \oplus v_{M} \cong v_{g}^{p} \cong h^{*} \tilde{\gamma}^{n-m} \oplus \varepsilon_{s}^{p-(n-m)}
$$

Como $m<n$, esse isomorfismo é equivalente a

$$
g^{*}\left(\tau_{N} \oplus v_{N}\right) \oplus \tau_{M} \oplus v_{M} \cong \tau_{M} \oplus g^{*} v_{N} \oplus h^{*} \tilde{\gamma}^{n-m} \oplus \varepsilon_{s}^{p-(n-m)},
$$

ou ainda,

$$
\varepsilon^{n+l} \oplus \varepsilon^{m+k} \cong \tau_{M} \oplus(g, h)^{*}\left(v_{N}^{l} \times \tilde{\gamma}^{n-m}\right) \oplus \varepsilon_{s}^{p-(n-m)}
$$

Usando novamente o fato que $m<n$, segue que

$$
\tau_{M} \oplus(g, h)^{*}\left(v_{N} \times \tilde{\gamma}^{n-m}\right) \cong \varepsilon^{m} \oplus \varepsilon^{l+(n-m)} .
$$

Seja $G$ o isomorfismo estável assim determinado.

Então, associaremos à classe $[M, g, s]$ dada, a classe da $\varphi$-variedade $(M,(g, h), G) \in \tilde{\rho}_{m}(N)$ determinada acima. 
Vamos mostrar que essa associação está bem definida.

Seja $\left(W^{m+1}, g, s\right)$ um bordismo entre as ternas $\left(M_{1}, g_{1}, s_{1}\right),\left(M_{2}, g_{2}, s_{2}\right)$ conforme ( 2.1 ). Pelo método descrito acima, essas ternas determinam a $\varphi$-variedade de dimensão $m+1\left(W^{m+1},(g, \tilde{h}), G\right)$ e as $\varphi$-variedades de dimensão $m\left(M_{1},\left(g_{1}, h_{1}\right), G_{1}\right)$, $\left(M_{2},\left(g_{2}, h_{2}\right), G_{2}\right)$, respectivamente.

Assim, $\tilde{h}$ e $G$ são obtidos a partir da decomposição de $v_{g}^{p}$ dada por $s$; digamos $g^{*} \tau_{N} \oplus v_{W} \cong \xi_{s}^{n-m} \oplus \varepsilon_{s}^{p-(n-m)}$.

Como $s_{\mid M_{1}}=s_{1}$, então $\quad\left(\varepsilon_{s}^{p-(n-m)}\right)_{\mid M_{1}}=\varepsilon_{s_{1}}^{p-(n-m)} \quad \mathrm{e}$, consequentemente, $\left(\xi_{s}^{n-m}\right)_{\mid M_{1}}=\xi_{s_{1}}^{n-m}$. Por outro lado, como $s_{\mid M_{2}}=\left(-s_{2}\right)$ ( no sentido de ( 2.1 ) ), então $\left(\varepsilon_{s}^{p-(n-m)}\right)_{\mid M_{2}}=-\varepsilon_{s_{2}}^{p-(n-m)}$, onde " - " indica uma inversão de sinal na componente de $\varepsilon_{s_{2}}^{p-(n-m)}$ fixada pela definição (2.1). Ainda assim, $\left(\xi_{s}^{n-m}\right)_{M_{2}}=\xi_{s_{2}}^{n-m}$.

A partir dessas considerações, podemos concluir que:

(1) $\tilde{h}_{\mid M_{i}}$ classifica $\xi_{s_{i}}$.

Segue então que $\tilde{h}_{\mid M_{i}}$ é homotópica a $h_{i}, i=1,2$. Mas, é possivel encontrar $h$ homotópica a $\tilde{h}$ tal que $h_{\mid M_{i}}=h_{i}, i=1,2$.

(2) $G_{\mid M_{1}}=G_{1}$ e $G_{\mid M_{2}}=-G_{2}$.

Isso mostra que $\left(W^{m+1},(g, h), G\right)$ è um bordismo entre $\left(M_{1},\left(g_{1}, h_{1}\right), G_{1}\right)$ e $\left(M_{2},\left(g_{2}, h_{2}\right), G_{2}\right)$.

Reciprocamente, seja dada a classe $[M,(g, h), G]$ de uma $\varphi$-variedade em $\tilde{d}_{m}(N)$. Lembramos que $g: M \rightarrow N, h: M \rightarrow B S O(n-m)$ são aplicações contínuas e $G$ é um isomorfismo estável de fibrados: $\tau_{M} \oplus(g, h)^{*}\left(v_{N}^{\prime} \times \tilde{\gamma}^{n-m}\right) \sim \varepsilon^{m} \oplus(g, h)^{*}\left(\varepsilon^{l+(n-m)}\right)$. Como em ( II.3 ), obtemos a partir de $G$ um isomorfismo de fibrados vetoriais

$$
v_{g}^{p}=g^{*} \tau_{N} \oplus v_{M} \cong h^{*} \gamma^{n-m} \oplus \varepsilon^{p-(n-m)} .
$$

Com essa decomposição de $v_{g}$, podemos concluir que $w_{1}\left(v_{g}\right)=0$ e determinar $p-(n-m)$ seções linearmente independentes de $v_{g} ;$ a saber, $s_{i}(x)=\left(0_{x}, e_{i}\right)$, onde $0_{x}$ é o vetor nulo na fibra sobre $x$ de $h^{*} \tilde{\gamma}^{n-m}$ e $e_{i}$ é o $i$-ésimo vetor da base canônica de $R^{p-(n-m)}$. Então, $s=\left(s_{1}, \ldots, s_{p-(n-m)}\right)$ é uma seção de $V_{p-(n-m)}\left(v_{g}^{p}\right)$. 
$\dot{A}$ classe $[M,(g, h), G] \in \tilde{\mathcal{I}}_{m}(N)$ dada, associaremos a terna $[M, g, s]$, onde $s$ é a seção de $V_{p-(n-m)}\left(v_{g}^{p}\right)$ determinada acima.

Vamos mostrar que essa associação está bem definida.

Seja $(W,(g, h), G)$ um bordismo entre as $\varphi$-variedades $\left(M_{1},\left(g_{1}, h_{1}\right), G_{1}\right)$, $\left(M_{2},\left(g_{2}, h_{2}\right), G_{2}\right)$. Pelo método descrito acima, a essas $\varphi$-variedades podemos associar ternas $(W, g, s),\left(M_{1}, g_{1}, s_{1}\right)$ e $\left(M_{2}, g_{2}, s_{2}\right)$, respectivamente.

Então, $s$ é obtida a partir da decomposição $v_{g}^{p} \cong h^{*} \tilde{\gamma}^{n-m} \oplus \varepsilon^{p-(n-m)}$ dada por $G$; a saber, $s_{i}(x)=\left(0_{x}, e_{i}\right)$, onde $0_{x}$ é o vetor nulo na fibra sobre $x \in W$ de $h^{*} \tilde{\gamma}^{n-m}$ e $e_{i}$ é o $i$-ésimo vetor da base canônica de $\mathbb{R}^{p-(n-m)}$, que è a fibra de $\varepsilon^{p-(n-m)}$ sobre $x \in W$. Como $G_{\mid M_{1}}=G_{1}$ e $G_{\mid M_{2}}=-G_{2}$, segue que $s_{\mid M_{1}}=s_{1}$ e $s_{\mid M_{2}}=\left(-s_{2}\right)$, no sentido de (2.1).

Assim, $(W, g, s)$ é um bordismo entre $\left(M_{1}, g_{1}, s_{1}\right)$ e $\left(M_{2}, g_{2}, s_{2}\right)$.

Portanto, existe uma bijeção entre $\tilde{\rho}_{m}(N)$ e o conjunto das classes de bordismo de ternas dadas pela definição ( 2.1 ).

2.3 Observação. Através da bijeção estabelecida na proposição ( 2.2 ), consideramos uma estrutura de grupo abeliano no conjunto das classes de bordismo definidas em ( 2.1 ). A partir de então tal grupo será identificado com $\tilde{\delta}_{m}(N)$.

2.4 Observação. Sem exigir $w_{1}\left(v_{g}\right)=0$, obtemos uma versão não orientada da Definição ( 2.1 ). De forma análoga à Proposição ( 2.2 ), podemos estabelecer uma estrutura de grupo abeliano no conjunto das classes de bordismo de ternas $(M, g, s)$ assim determinadas, através de uma bijeção com o grupo de bordismo normal $\Omega_{m}(N)=\Omega_{m}\left(N \times B O(n-m) ; \varphi_{n-m}\right)$, onde $\varphi_{n-m}=\varepsilon^{1+(n-m)}-v_{N}^{l} \times \gamma^{n-m}$ é fibrado virtual sobre $N \times B O(n-m) \quad$ (Ver II.3 ). Os dois grupos são então identificados. 


\section{A função $l_{f}$}

Seja $f: M^{m} \rightarrow N^{n}, m<n$, uma aplicação homotópica a uma imersão. Então, $f \dot{e}$ bordante a uma imersão. Se $w_{1}\left(v_{f}\right)=0$, então $[M, f]$ pertence à imagem do homomorfismo de "esquecimento"

$$
\tilde{\mathcal{F}}_{m}: \tilde{d}_{m}(N) \rightarrow \Omega_{m}(N)
$$

Caso contrário, trata-se da versão não orientada e $[M, f]$ está na imagem do homomorfismo de "esquecimento"

$$
\mathcal{F}_{m}: \mathcal{d}_{m}(N) \rightarrow \eta_{m}(N)
$$

Vamos estudar os dois casos simultaneamente, adotando a notação sem " ".

Em termos da interpretação de $\ell_{m}(N)$ obtida em ( 2.3 e 2.4 ), esse homomorfismo é dado por

$$
\mathcal{F}_{m}\left(\left[M^{\prime}, f^{\prime}, s^{\prime}\right]\right)=\left[M^{\prime}, f^{\prime}\right]
$$

Vamos denotar por $\mathcal{C}_{f}$ a imagem inversa de $[M, f]$ por $\mathscr{F}_{m}$. Tal conjunto é então constituído pelas classes de bordismo normal de aplicações homotópicas a imersões que são bordantes a $f$. Observamos ainda que $\mathcal{C}_{f}$ nada mais é que a classe lateral $\operatorname{ker} \mathcal{F}_{m}+\alpha$, onde $\mathscr{F}_{m}(\alpha)=[M, f]$. Portanto, $C_{f}$ e ker $\mathscr{F}_{m}$ possuem a mesma cardinalidade.

A fim de comparar $I[M, N]_{f}$ e $\mathcal{C}_{f}$, como proposto na introdução desse capitulo, definimos a seguinte função:

$$
3.1 \ell_{f}: V_{f} \rightarrow \ell_{f} \text { dada por } \ell_{f}([s])=[M, f, s] \text {. }
$$

Vamos primeiramente analisar se $\ell_{f}$ está bem definida. Sejam $s_{0}, s_{1}$ seções homotópicas de $V_{p-(n-m)}\left(v_{f}\right)$. Então, $s_{0}$ e $\left(-s_{1}\right)$ também são homotópicas; assim, existe $H: M \times I \rightarrow E\left(V_{p-(n-m)}\left(v_{f}\right)\right)$ tal que $H(x, 0)=s_{0}(x), H(x, 1)=\left(-s_{1}\right)(x)$ e $H_{t}$ é uma seção de $V_{p-(n-m)}\left(v_{f}\right)$ para todo $t \in I$. 
Tomemos $W=M \times I$ e $g: W \rightarrow N$ dada por $g(x, t)=f(x)$; ou seja, $g=f \circ \pi_{1}$. Então, como $v_{M \times l} \cong \pi_{i}^{*} v_{M}$, temos $v_{g} \cong \pi_{i}^{*} v_{f} \quad$ e, consequentemente, $V_{p-(n-m)}\left(v_{g}\right) \cong \pi_{1}^{*}\left(V_{p-(n-m)}\left(v_{f}\right)\right)$.

Assim, $s: M \times I \rightarrow E\left(V_{p-(n-m)}\left(v_{g}\right)\right)$ dada por $s((x, t))=((x, t), H(x, t))$ define uma seção de $V_{p-(n-m)}\left(v_{g}\right)$ tal que $s_{\mid M \times\{0\}}=s_{0}$ e $s_{\mid M \times\{1\}}=s_{1}$.

Portanto, $(M \times I, g, s)$ determina um bordismo entre $\left(M, g, s_{0}\right)$ e $\left(M, g, s_{1}\right)$. Isso mostra que $l_{f}$ está bem definida.

3.2 Observação. $O$ diagrama abaixo

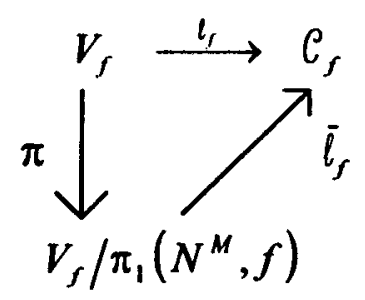

é comutativo, onde $\bar{l}_{f}(\{[s]\})=[M, f, s]$ e $\pi$ é a projeção natural. De fato, consideremos $\left[s_{0}\right],\left[s_{1}\right]=\left[-s_{1}\right]$ elementos de $V_{f}$, pertencentes a mesma órbita pela ação de $\pi_{1}\left(N^{M}, f\right)$. Então, por ( 1.2), existe uma homotopia $F: M \times I \rightarrow N$ entre $f$ e $f$, e uma seção $s$ de $V_{p-(n-m)}\left(v_{F}\right)$ tal que $s_{\mid M \times\{0\}}=s_{0}$ e $s_{\mid M \times\{1\}}=\left(-s_{1}\right)$. Isso mostra que $(M \times I, F, s)$ é um bordismo entre $\left(M, f, s_{0}\right)$ e $\left(M, f, s_{1}\right)$.

Portanto, a menos da bijeção $V_{f} / \pi_{1}\left(N^{M}, f\right) \leftrightarrow I[M, N]_{f}(1.3)$, a função $\bar{l}_{f}$ determina uma função de $I[M, N]_{f}$ em $\mathcal{C}_{f}$.

3.3 Observação. A função $\ell_{f}$ pode não ser injetora. Por exemplo, consideremos $f: M^{m} \rightarrow N^{2 m-1}$; então, $f$ é homotópica a uma imersão. No caso em que $M, N$ são orientáveis, com $f^{\bullet}\left(w_{2}(N)\right)=0$, Li Banghe prova em [ 14] que, para $m \equiv 1 \bmod 4$,

$$
V_{f} \leftrightarrow H_{1}(M ; \mathrm{Z}) \oplus \mathrm{Z}_{2} \oplus \mathrm{Z}_{2},
$$


onde "↔" denota bijeção. Se $N$ for uma $\pi$-variedade, mostramos que $K_{m}^{1} \stackrel{e_{2}}{\cong} H_{1}(N ; Z)$ ( T. II.3.3 ). Então, se $H_{1}(N ; \mathrm{Z})=0$, temos que $K_{m}^{1} \in C_{2}$ e, portanto, é finito. Segue então que $\mathcal{C}_{f}$ também é finito. Assim, para $M$ tal que $H_{1}(M ; Z)$ é infinito, a função $l_{f}$ não é injetora.

A função $l_{f}$ pode não ser sobrejetora. Isso ocorre, por exemplo, se tivermos $H_{1}(N ; Z)$ infinito e $H_{1}(M ; Z)$ finito, com $M$ e $N$ nas condições anteriores.

\section{Alguns resultados sobre a imagem de $\ell_{f}$}

$O$ estudo da imagem de $\ell_{f}$ envolve questões sobre a extensão de seções de fibrados. As técnicas usadas encontram-se na teoria de obstrução, particularmente aquelas introduzidas por Liao em [ 11 ]. Faremos a seguir um breve resumo dos resultados desse artigo que serão utilizados nesse parágrafo.

Seja $B: E \stackrel{p}{\longrightarrow} B$ um fibrado com fibra $F$, complexo $C W$ finito $(q-1)$ conexo, $q \geq 2$, e espaço base $B$, complexo $C W$ conexo. Consideremos a ação de $\pi_{1}(B)$ em $\pi_{q}(F)$ trivial.

Suponhamos que o fibrado $B$ possua uma seção $s$ sobre o $(q+1)$-esqueleto de $B, \quad B^{q+1}$. $\quad$ A classe de homotopia de $s$ restrita a $B^{q}$ é associada uma classe de cohomologia $\theta(s) \in H^{q}\left(E ; \pi_{q}(F)\right)$, conforme descrevemos a seguir. É provado que existe um elemento $e \in H^{q}\left(E ; \pi_{q}(F)\right)$ tal que $i^{*} e=k$, onde $i^{*}$ é a induzida em cohomologia pela inclusão $i: F \rightarrow E$ e $k \in H^{q}\left(F ; \pi_{q}(F)\right)$ é a obstrução primária à contração de $F$ a um ponto. Então, $\theta(s)$ é definido por:

4.1 $\theta(s)=e-p^{*} s^{*} e \in H^{q}\left(E ; \pi_{q}(F)\right)$. 
( O homomorfismo dual $\quad s^{*}: H^{q}\left(E ; \pi_{q}(F)\right) \rightarrow H^{q}\left(B ; \pi_{q}(F)\right.$ ) está definido porque $s$ é uma seção sobre o $(q+1)$-esqueleto de $B)$.

A classe $\theta(s)$ independe da escolha de $e$ e é o único elemento de $H^{q}\left(E ; \pi_{q}(F)\right)$ que satisfaz as condições: $s^{*} \theta(s)=0$ e $i^{*} \theta(s)=k$. Além disso, as seguintes propriedades são válidas:

(i) Duas seções $s_{1}, s_{2}$ de $\beta$ sobre $B^{q+1}$ são homotópicas sobre o $q$-esqueleto de $B$ se, e somente se, $\theta\left(s_{1}\right)=\theta\left(s_{2}\right)$.

(ii ) Se $r$ é outra seção de $B$ sobre $B^{q+1}$, então $\theta(r)=\theta(s)-p^{*} r^{*} \theta(s)$. Consequentemente, $s^{*} \theta(r)=-r^{*} \theta(s)$.

(iii ) Considere fixada uma seção $r$ de $\beta$ sobre $B^{q+1}$. Então, para quaisquer seções $s_{1}, s_{2}$ de $B$ sobre $B^{q+1}$,

$$
\theta\left(s_{1}\right)^{j}=\theta\left(s_{2}\right) \text { se, e somente se, } s_{1}^{*}(\theta(r))=s_{2}^{*}(\theta(r)) \text {. }
$$

(iv) Para cada $e \in H^{q}\left(E ; \pi_{q}(F)\right)$ tal que $i^{*} e=k$, existe uma seção $s$ de $\beta$ sobre $B^{q+1}$ tal que $\theta(s)=e$.

( v ) Sejam $s_{1}, s_{2}$ seções de $B$ sobre $B^{q+1}$ e $\bar{d}\left(s_{1}, s_{2}\right) \in H^{q}\left(B ; \pi_{q}(F)\right)$ sua diferença primária. Então,

$$
p^{*}\left(\bar{d}\left(s_{1}, s_{2}\right)\right)=\theta\left(s_{1}\right)-\theta\left(s_{2}\right) .
$$

A partir dessas propriedades prova-se o seguinte

4.2 Teorema. Seja $B: E \stackrel{p}{\longrightarrow} B$ um fibrado com fibra $F$, complexo $C W$ finito $(q-1)$-conexo, $q \geq 2$, e espaço base $B$, complexo $C W$ conexo. Consideremos a ação de $\pi_{1}(B)$ em $\pi_{q}(F)$ trivial. Suponhamos que $B$ possua uma seção $r$ sobre $B^{q+1}$.

Então, a função

$$
\begin{aligned}
\left\{[s]_{q} ; \text { é seção de } \beta \text { sobre } B^{q+1}\right\} & \rightarrow H^{q}\left(B ; \pi_{q}(F)\right) \\
{[s]_{q} } & \mapsto s^{*}(\theta(r))
\end{aligned}
$$

é bijetora. 
Apresentamos a seguir os resultados que obtivemos sobre a imagem de $l_{f}$. Primeiramente vamos estabelecer a notação usada.

Para uma aplicação $g: M^{m} \rightarrow N^{n}$ denotamos por $\beta_{g}$ o fibrado de frames de codimensão $n$ - $m$ do fibrado normal estável de $g$, que consideramos com posto $p$ suficientemente grande.

4.3 Seja $f: M^{m} \rightarrow N^{2 m-1}$ uma aplicação fixada e seja $\left[M^{\prime}, f^{\prime}, r^{\prime}\right]$ um elemento de $C_{f}, m \geq 3$. Suponhamos que exista um bordismo $(W, g, R)$ entre $\left(M^{\prime}, f^{\prime}, r^{\prime}\right)$ e $(M, f, r)$, onde $r=R_{\left.\right|_{M}}$. Os fibrados $B_{g}, B_{f}$ e $B_{f}$. possuem como fibra a variedade de Stiefel $F=V_{p-(m-1)}\left(R^{p}\right)$, que é um complexo $C W$ finito $(m-2)$-conexo. Analisemos a ação do grupo fundamental dos respectivos espaços base em $\pi_{m-1}(F)$. Lembramos que esse grupo é isomorfo a $Z_{2}$, se $m$ for par, e isomorfo a $Z$, se $m$ for impar [26,25.6]. Assim, para $m$ par, tal ação é sempre trivial. No caso $m$ ímpar, vamos supor que tal fato ocorra e que os espaços base sejam variedades orientadas. Então, pelo Teorema ( 4.2 ), segue que as funções abaixo são bijetoras:

$$
\begin{aligned}
\left\{[S]_{m-1} ; S \text { é seção de } B_{g} \text { sobre } W^{m}\right\} & \rightarrow H^{m-1}\left(W ; \pi_{m-1}(F)\right) \\
{[S]_{m-1} } & \mapsto S(\theta(R)) \\
\left\{[s]_{m-1} ; s \text { é seção de } B_{f} \text { sobre } M\right\} & \rightarrow H^{m-1}\left(M ; \pi_{m-1}(F)\right) \\
{[s]_{m-1} } & \mapsto s^{*}(\theta(r)) \\
\left\{\left[s^{\prime}\right]_{m-1} ; s^{\prime} \text { é seção de } B_{f} \text { sobre } M^{\prime}\right\} & \rightarrow H^{m-1}\left(M^{\prime} ; \pi_{m-1}(F)\right) \\
{\left[s^{\prime}\right]_{m-1} } & \mapsto s^{*}(\theta(r))
\end{aligned}
$$

4.4 Proposição. Seja $\left[M^{\prime}, f^{\prime}, r^{\prime}\right]$ um elemento da imagem de $l_{f}$. Seja $(W, g, R)$ um bordismo entre $\left(M^{\prime}, f^{\prime}, r^{\prime}\right)$ e $(M, f, r), r=R_{l_{M}}$.

Suponhamos que para todo elemento da forma $\left[M^{\prime}, f^{\prime}, s^{\prime}\right] \in \mathcal{C}_{\boldsymbol{f}}$ exista uma seção $S$ de $B_{g}$ de modo que $(W, g, S)$ seja um bordismo entre $\left(M^{\prime}, f^{\prime}, s^{\prime}\right)$ e $(M, f, s)$, 
$s=S_{\mid M} . \quad$ Então, $\quad i_{*}^{\prime}\left(H_{1}\left(M^{\prime} ; \pi_{m-1}(F)\right)\right) \subset i_{*}\left(H_{1}\left(M ; \pi_{m-1}(F)\right)\right)$, onde $\quad i^{\prime}: M^{\prime} \rightarrow W$, $i: M \rightarrow W$ são inclusões.

Demonstração. Tomemos $u^{\prime} \in H_{1}\left(M^{\prime} ; \pi_{m-1}(F)\right)$ e consideremos sua classe dual $D_{M}^{-1}\left(u^{\prime}\right) \in H^{m-1}\left(M^{\prime} ; \pi_{m-1}(F)\right)$. Pela bijeção estabelecida em ( 4.3 ) é possivel determinar uma seção $s^{\prime}$ de $B_{f}$, de modo que $s^{\prime *}\left(\theta\left(r^{\prime}\right)\right)=D_{M^{\prime}}^{-1}\left(u^{\prime}\right)$.

Dado o elemento $\left[M^{\prime}, f^{\prime}, s^{\prime}\right] \in \mathcal{C}_{f}$, segue pela hipótese que existe uma seção $S$ de $B_{g}$ tal que $(W, g, S)$ seja um bordismo entre $\left(M^{\prime}, f^{\prime}, s^{\prime}\right)$ e $(M, f, s), \quad s=S_{\mid M}$. Novamente por ( 4.3 ), à classe de homotopia $[S]_{m-1}$ corresponde uma única classe de cohomologia $S^{*}(\theta(R)) \in H^{m-1}\left(W ; \pi_{m-1}(F)\right)$.

Seja $\alpha \in H_{2}\left(W, M \cup M^{\prime} ; \pi_{m-1}(F)\right)$ a classe de homologia dual a $S^{*}(\theta(R))$, ou seja, $\alpha=D_{w}\left(S^{*}(\theta(R))\right)$. Então, pelo diagrama comutativo

$$
\begin{gathered}
\ldots \rightarrow H^{m-1}\left(W ; \pi_{m-1}(F)\right) \stackrel{\left(i^{*}, r^{*}\right)}{\longrightarrow} H^{m-1}\left(M ; \pi_{m-1}(F)\right) \oplus H^{m-1}\left(M^{\prime} ; \pi_{m-1}(F)\right) \rightarrow \ldots \\
\downarrow \\
\downarrow D_{W} \\
\ldots \rightarrow D_{2}\left(W ; M \cup D_{M^{\prime}}\right.
\end{gathered}
$$

temos que

$$
\begin{gathered}
\partial \alpha=\partial D_{W}\left(S^{*}(\theta(R))\right)=\left(D_{M}+D_{M^{*}}\right)\left(i^{*}, i^{*}\right)\left(S^{*}(\theta(R))\right)= \\
\left(D_{M}+D_{M^{\prime}}\right)\left(i^{*}\left(s^{*}(\theta(R))\right), i^{*}\left(S^{*}(\theta(R))\right)\right)= \\
\left(D_{M}+D_{M^{\prime}}\right)\left(s^{*}(\theta(r))+s^{* *}\left(\theta\left(r^{\prime}\right)\right)\right)=D_{M}\left(s^{*}(\theta(r))\right)+u^{\prime}
\end{gathered}
$$

Seja $u=D_{M}\left(s^{*}(\theta(r))\right) \in H_{1}\left(M ; \pi_{m-1}(F)\right) . \quad$ Então, $\quad \partial \alpha=u+u^{\prime} \quad$ e, consequentemente, $\cdot\left(i_{*}+i_{*}^{\prime}\right)\left(u+u^{\prime}\right)=0 . \quad$ Portanto, $\quad i_{*}^{\prime}\left(u^{\prime}\right)=i_{*}(-u), \quad$ com $-u \in H_{1}\left(M ; \pi_{m-1}(F)\right)$.

No caso $m$ par há uma reciproca desse resultado. Necessitamos do seguinte 
4.5 Lema. Seja $f: M^{m} \rightarrow N^{2 m-1}$ uma aplicação contínua, com $m$ par. Para $m \equiv 2 \bmod 4$ suponhamos que $f^{*} w_{2}(N)+f^{*} w_{1}(N) \cdot w_{1}(M) \neq 0$.

Então, existe uma bijeção entre $V_{f}$ e o conjunto das classes de homotopia sobre $M^{m-1}$ de seções de $B_{f}$.

Demonstração. Com essas hipóteses, Li Banghe prova em [ 14 ] que

$$
V_{f} \leftrightarrow H^{m-1}\left(M ; Z_{2}\right)
$$

Por outro lado, por ( 4.3 ), temos que

$$
\left\{[s]_{m-1} ; s \text { é seção de } \beta_{f}\right\} \leftrightarrow H^{m-1}\left(M ; Z_{2}\right),
$$

donde segue o lema.

4.6 Proposição. Seja $m$ um inteiro positivo par. Seja $\left[M^{\prime}, f^{\prime}, r^{\prime}\right]$ um elemento da imagem de $\ell_{f}$. Se $m \equiv 2 \bmod 4$, suponhamos $f^{\prime \prime} w_{2}(N)+f^{\prime *} w_{1}(N) \cdot w_{1}\left(M^{\prime}\right) \neq 0$.

Seja $(W, g, R)$ um bordismo entre $\left(M^{\prime}, f^{\prime}, r^{\prime}\right)$ e $(M, f, r), r=R_{M M}$.

Se $i_{*}^{\prime}\left(H_{1}\left(M^{\prime} ; Z_{2}\right)\right) \subset i_{*}\left(H_{1}\left(M ; Z_{2}\right)\right)$, então para todo elemento da forma $\left[M^{\prime}, f^{\prime}, s^{\prime}\right] \in \mathcal{C}_{f}$, existe uma seção $S$ de $\beta_{g}$ tal que $(W, g, S)$ seja um bordismo entre $\left(M^{\prime}, f^{\prime}, s^{\prime}\right)$ e $(M, f, s), s=S_{\mid M}$.

Demonstração. Seja dado um elemento da forma $\left[M^{\prime}, f^{\prime}, s^{\prime}\right]$ em $C_{f}$. Por ( 4.3 ), à classe de homotopia $\left[s^{\prime}\right]_{m-1}$ corresponde uma única classe de cohomologia em $H^{m-1}\left(M^{\prime} ; Z_{2}\right)$; a saber, $\quad s^{\prime *}\left(\theta\left(r^{\prime}\right)\right)$. Seja $u^{\prime}=D_{M^{\prime}}\left(s^{\prime *}\left(\theta\left(r^{\prime}\right)\right)\right)$ sua classe dual em $H_{1}\left(M^{\prime} ; Z_{2}\right)$. Pela hipótese, existe $(-u) \in H_{1}\left(M ; Z_{2}\right)$ tal que $i_{:}^{\prime}\left(u^{\prime}\right)=i_{*}(-u)$ e, então, $\left(i_{*}+i_{*}^{\prime}\right)\left(u+u^{\prime}\right)=0$. Pela exatidão da sequência de homologia do par $\left(W, M \cup M^{\prime}\right)$, existe $\alpha \in H_{2}\left(W, M \cup M^{\prime} ; Z_{2}\right) \quad$ tal que $\quad \partial \alpha=u+u^{\prime}$. 
Novamente por ( 4.3 ), à classe dual de $\alpha$ está associada uma única classe de homotopia $\left[S^{\prime}\right]_{m-1}$, onde $S^{\prime}$ é uma seção de $B_{g}$ sobre $W^{m}$, de modo que $\alpha=D_{W}\left(S^{\prime} \cdot(\theta(R))\right)$. Como $H^{m+1}\left(W ; Z_{2}\right)=0, \quad S^{\prime}$ se estende a uma seçâo $\bar{S}$ de $B_{g}$ e temos $\bar{S}^{*}(\theta(R))=S^{* *}(\theta(R))$. Da comutatividade do diagrama

$$
\begin{gathered}
\ldots \rightarrow H^{m-1}\left(W ; Z_{2}\right) \stackrel{\left(i \cdot i^{*}\right)}{\longrightarrow} H^{m-1}\left(M ; Z_{2}\right) \oplus H^{m-1}\left(M^{\prime} ; Z_{2}\right) \rightarrow \ldots \\
\\
\downarrow
\end{gathered}
$$

segue que $u^{\prime}=D_{M^{\prime}}\left(\left(\bar{S}_{\mid M^{\prime}}\right)^{*}\left(\theta\left(r^{\prime}\right)\right)\right)$. Então, $s^{\prime^{*}}\left(\theta\left(r^{\prime}\right)\right)=\left(\bar{S}_{\mid M^{\prime}}\right)^{*}\left(\theta\left(r^{\prime}\right)\right) ;$ logo, por ( 4.3$)$, $\left[s^{\prime}\right]_{m-1}=\left[\bar{S}_{\mid M^{\prime}}\right]_{m-1}$. Pelo Lema ( 4.5$)$, segue que $s^{\prime}$ é homotópica a $\bar{S}_{\mid M^{\prime}}$. Como $\bar{S}_{\mid M^{\prime}}$ se estende a uma seção $\bar{S}$ de $B_{\boldsymbol{g}}$, segue que $s^{\prime}$ também se estende a uma seção $S$ de $B_{\boldsymbol{g}}$. Então, $(W, g, S)$ é um bordismo entre $\left(M^{\prime}, f^{\prime}, s^{\prime}\right)$ e $(M, f, s), s=S_{\mid M}$.

4.7 Observação. A Proposição ( 4.6 ) nos fornece uma técnica para determinar seções bordantes que não sejam homotópicas. Consideremos as mesmas hipóteses da referida proposição e tomemos $m \equiv 2 \bmod 4$. Dado $u^{\prime} \in H_{1}\left(M^{\prime} ; Z_{2}\right)$, se $u \in H_{1}\left(M ; Z_{2}\right)$ é tal que $i_{*}^{\prime}\left(u^{\prime}\right)=i_{*}(u)$, então para todo $v \in \operatorname{ker} i_{*}$, temos $i_{*}^{\prime}\left(u^{\prime}\right)=i_{*}(u+v)$. Como na demonstração anterior, é possível determinar $\alpha_{v} \in H_{2}\left(W, M \cup M^{\prime} ; Z_{2}\right)$ tal que $\partial \alpha_{v}=u^{\prime}-(u+v)$ e, por (4.3), encontrar $S_{v}$ seção de $B_{g}$ tal que $\alpha_{v}=D_{W}\left(S_{v}^{*}(\theta(R))\right)$.

Denotemos $S_{v \mid M^{\prime}}$ por $s_{v}^{\prime}$ e, no caso particular de $v=0$, por $s^{\prime}$. Então, $D_{M^{\prime}}\left(s_{v}^{\prime^{*}}\left(\theta\left(r^{\prime}\right)\right)\right)=u^{\prime}=D_{M^{\prime}}\left(s^{\prime *}\left(\theta\left(r^{\prime}\right)\right)\right)$. Consequentemente, $\left[s_{v}^{\prime}\right]_{m-1}=\left[s^{\prime}\right]_{m-1}$. Como a variedade $M^{\prime}$ satisfaz as hipóteses do Lema ( 4.5 ), segue que $\left[s_{v}^{\prime}\right]=\left[s^{\prime}\right]$ e, portanto, $\left(M^{\prime}, f^{\prime}, s_{v}^{\prime}\right)$ é bordante a $\left(M^{\prime}, f^{\prime}, s^{\prime}\right)$.

Por outro lado, podemos considerar as seções $s_{v}=S_{v \mid M}$ de $\beta_{f}, v \in \operatorname{ker} i_{*}$. Como $\left(M, f, s_{v}\right)$ é bordante a $\left(M^{\prime}, f^{\prime}, s_{v}^{\prime}\right)$ e $(M, f, s)$ é bordante a $\left(M^{\prime}, f^{\prime}, s^{\prime}\right)$, segue que 
$\left(M, f, s_{v}\right)$ é bordante a $(M, f, s)$. Então, $\ell_{f}\left(\left[s_{v}\right]\right)=\ell_{f}([s])$, para todo $v \in$ ker $i_{\odot}$. Mas, $l_{f}$ pode não ser injetora. Assim, as seções $s_{v}$ de $B_{f}, v \in$ ker $i_{*}$, não são necessariamente homotópicas.

4.8 Proposição. Seja $N$ variedade $n$-dimensional, $q$-simples para $q \leq m$ e tal que o homomorfismo de Hurewicz $\mathcal{H}: \pi_{m}(N) \rightarrow H_{m}(N ; Z)$ seja injetor.

Se $(M, f)$ é bordante a $\left(M^{\prime}, f^{\prime}\right)$, é possivel determinar um bordismo $(W, g)$ entre eles de modo que o homomorfismo induzido $g_{\#}: \pi_{1}(W) \rightarrow \pi_{1}(N)$ seja injetor.

Demonstração. Seja $\left(W^{\prime}, g^{\prime}\right)$ um bordismo entre $(M, f)$ e $\left(M^{\prime}, f^{\prime}\right)$.

Consideremos $\lambda: S^{\prime} \rightarrow W^{\prime}$ um gerador de $\operatorname{ker} g_{\#}^{\prime}$ e $h: S^{1} \times D^{m} \rightarrow W^{\prime}$ um mergulho que represente $\lambda$. Tomemos $W=\left[W^{\prime}-h\left(S^{1} \times 0\right)\right]+D^{2} \times S^{m-1} / \equiv$, onde a relação $\equiv$ identifica $h(u, t v)$ com $(t u, v), u \in S^{\prime}, v \in S^{m-1}$, ou seja, $W$ é obtido de $W^{\prime}$ por uma cirurgia do tipo $(1, m)$. O grupo fundamental $\pi_{1}(W)$ é isomorfo ao quociente de $\pi_{1}\left(W^{\prime}\right)$ por um subgrupo que contém $\lambda[21]$.

Analisemos a possibilidade de estender $\boldsymbol{g}^{\prime}$ a uma aplicação $g$ definida em $W$. Para tanto, basta verificar se $G=g_{\mid s^{1} \times S^{-1}}^{\prime}: S^{1} \times S^{m-1} \rightarrow N$ se estende a $D^{2} \times S^{m-1}$. Sendo $D^{2} \times S^{m-1}$ um espaço conexo por caminhos, é possível estender $G$ ao 1-esqueleto de $D^{2} \times S^{m-1}$. Quanto à extensão ao 2-esqueleto de $D^{2} \times S^{m-1}$,basta observar que $\pi_{1}\left(D^{2} \times S^{m-1}\right)=0$ e que $G_{t i}\left(\left[e_{1}\right]\right)=0$, onde $\left[e_{1}\right]$ é um gerador de $\pi_{1}\left(S^{1} \times S^{m-1}\right)$. Também podemos estender $G$ ao $q$-esqueleto de $\quad D^{2} \times S^{m-1}$, para $3 \leq q \leq m$, pois a obstrução à extensão é uma classe de cohomologia em $H^{q}\left(D^{2} \times S^{m-1}, S^{1} \times S^{m-1} ; \pi_{q-1}(N)\right)$ que é nulo. 
Uma vez que $H^{m+1}\left(D^{2} \times S^{m-1}, S^{1} \times S^{m-1} ; \pi_{m}(N)\right) \cong \pi_{m}(N)$, analisaremos a extensão a $D^{2} \times S^{m-1}$ através do seguinte processo. Retiramos de $D^{2} \times S^{m-1}$ um disco fechado $D=D^{m+1}$ e observamos que $H^{m+1}\left(D^{2} \times S^{m-1}-\dot{D}, S^{1} \times S^{m-1} ; \pi_{m}(N)\right)=0$, visto que $D^{2} \times S^{m-1}-\stackrel{\circ}{D}$ é uma variedade aberta, com bordo $S^{1} \times S^{m-1} \cup S^{m}$. Então, é possível estender $G$ a $D^{2} \times S^{m-1}-\dot{D}$. Seja $k: S^{m} \rightarrow N$ a restrição dessa extensão ao bordo de $D$. Basta, então, verificar se $k$ se estende ao interior de $D$, ou equivalentemente, se $k$ é homotópica a uma constante. Tomemos $\tilde{W}$ a variedade obtida pela união disjunta de $W^{\prime}$ e $W-\stackrel{D}{D}$, identificando pelo bordo comum $M \cup M^{\prime}$. Então, $\partial \tilde{W}=S^{m}$.

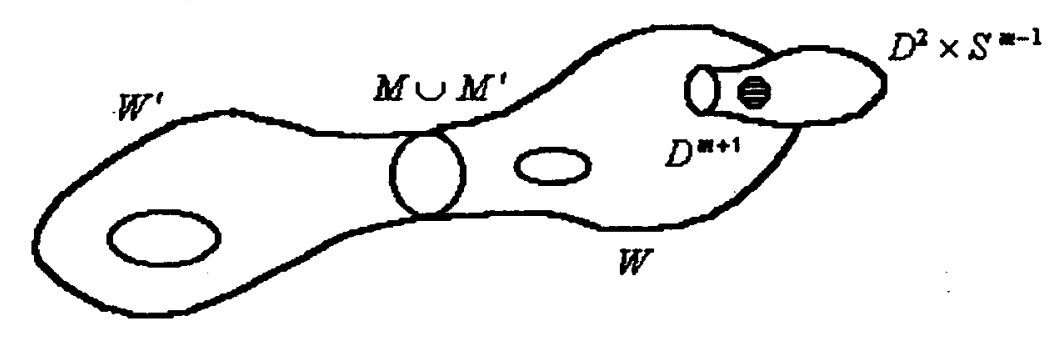

Seja $\mu_{\tilde{W^{\prime}}} \in H^{m+1}\left(\tilde{W}, S^{m}\right)$ a classe fundamental de homologia. Se $\tilde{g}$ é a extensão de g a $\tilde{W}$ e $\quad i: S^{m} \rightarrow \tilde{W} \quad$ é a inclusão, então $k_{*}\left(\mu_{s^{*}}\right)=(\tilde{F} \circ i),\left(\mu_{s^{*}}\right)=\tilde{F}_{*} \circ i_{*}\left(\partial \mu_{\tilde{W}}\right)=$ $\tilde{F}_{*}\left(i_{*} \partial\left(\mu_{\tilde{W}}\right)\right)=\tilde{F}_{*}(0)=0$. Sendo $\mathcal{H}: \pi_{m}(N) \rightarrow H_{m}(N ; \mathrm{Z})$ um monomorfismo e $\mathcal{H}([k])=k_{*}\left(\mu_{s^{m}}\right)=0$, obtemos $[k]=0$, donde segue o resultado.

Repetindo esse processo para cada gerador de $\operatorname{ker} g_{\sharp}^{\prime}$, obtemos um bordismo $(W, g)$ entre $(M, f)$ e $\left(M^{\prime}, f^{\prime}\right)$ de modo que $g_{\sharp}: \pi_{1}(W) \rightarrow \pi_{1}(N)$ seja injetor.

4.9 Corolário. Seja $N$ variedade $n$-dimensional, satisfazendo as hipóteses da proposição anterior. Seja $\left[M^{\prime}, f^{\prime}, s^{\prime}\right]$ um elemento da imagem de $\ell_{f}$. Então, existe um 
bordismo $(W, g, s)$ entre $\left(M^{\prime}, f^{\prime}, s^{\prime}\right)$ e $(M, f, s), s=S_{\mid M}$, de modo que o homomorfismo induzido $g_{\#}: \pi_{1}(W) \rightarrow \pi_{1}(N)$ seja injetor.

Demonstração. Seja $\left[M^{\prime}, f^{\prime}, s^{\prime}\right]$ um elemento da imagem de $\ell_{f}$. Então, existe um bordismo $\left(W^{\prime}, g^{\prime}, R^{\prime}\right)$ entre $\left(M^{\prime}, f^{\prime}, s^{\prime}\right)$ e $\left(M, f, R_{M}^{\prime}\right)$. Em particular, $\left(W^{\prime}, g^{\prime}\right)$ é um bordismo entre $(M, f)$ e $\left(M^{\prime}, f^{\prime}\right)$. Pela proposição anterior, podemos substituir $\left(W^{\prime}, g^{\prime}\right)$ por um bordismo $(W, g)$ de modo que $g_{\#}: \pi_{1}(W) \rightarrow \pi_{1}(N)$ seja monomorfismo. Retomando a demonstração da proposição anterior, analisemos a possibilidade de estender $R^{\prime}$ a uma seção $R$ de $B_{g}$ definida em $W$. Para tanto, basta verificar se $R_{\mid s^{1} \times s^{m-1}}^{\prime}$ se estende a $D^{2} \times S^{m-1}$. A fibra $L$ de $B_{g} \dot{e} \quad(m-2)$-conexa; portanto, existe extensão de $R_{\mid S^{1} \times S^{m-1}}^{\prime}$ até o $(m-1)$-esqueleto de $D^{2} \times S^{m-1}$. Também podemos estendê-la ao m-esqueleto de $D^{2} \times S^{m-1}$, pois sua obstrução pertence a $H^{m}\left(D^{2} \times S^{m-1}, S^{1} \times S^{m-1} ; \pi_{m-1}(L)\right)$ que é nulo.

Analisemos a extensão ao $(m+1)$-esqueleto de $D^{2} \times S^{m-1}$. Retiremos um disco fechado $D=D^{m+1}$ de $D^{2} \times S^{m-1}$ : uma vez que $H^{m+1}\left(D^{2} \times S^{m-1}-\dot{D}, S^{1} \times S^{m-1} ; \pi_{m}(L)\right)=0$, existe uma extensão a $D^{2} \times S^{m-1}-\dot{D}$. Dessa forma, obtemos uma seção $R$ de $B_{g}$ sobre $W-\stackrel{\circ}{D}$, que contém o $m$-esqueleto de $W$. Sendo $\quad r^{\prime}=R_{\mathcal{M}^{\prime}}^{\prime}=R_{\left.\right|_{M}}$, a seção $R$ é uma extensão de $\quad r^{\prime}$ a $W-\dot{D}$ e, em particular, $R_{W^{*}}$ é uma extensão de $r^{\prime}$ a $W^{m}$. A obstrução à extensão de $R_{W^{m}}$ ao $(m+1)$-esqueleto de $W$ pertence a $H^{m+1}\left(W, M^{\prime}\right)=0$; portanto, $R_{\mid w^{\prime \prime}}$ se estende a uma seção $S$ de $B_{g}$.

Obtemos, então, $\left(M^{\prime}, f^{\prime}, s^{\prime}\right)$ bordante a $(M, f, s), s=S_{\mid M}$, através do bordismo $(W, g, S)$, onde $g_{\#}: \pi_{1}(W) \rightarrow \pi_{1}(N)$ é um monomorfismo. 
4.10 Corolário. Seja $N$ variedade $(2 m-1)$-dimensional, 1-conexa e tal que o homomorfismo de Hurewicz $\quad \mathcal{H}: \pi_{m}(N) \rightarrow H_{m}(N ; \mathrm{Z})$ seja injetor. Então, a função $\ell_{f}$ é sobrejetora.

Demonstração. Seja $\left[M^{\prime}, f^{\prime}, s^{\prime}\right] \in \mathcal{C}_{f}$. Então, $\left(M^{\prime}, f^{\prime}\right)$ é bordante a $(M, f)$. Pela Proposição ( 4.9 ), é possivel determinar um bordismo $(W, g)$ entre eles de modo que $g_{\#}: \pi_{1}(W) \rightarrow \pi_{1}(N)$ seja monomorfismo. Segue desse fato e da hipótese $N$ 1-conexa que $\pi_{1}(W)=0$.

Como a fibra de $\beta_{g}$ é $(m-2)$-conexa, é possivel estender $s^{\prime}$, que é uma seção de $\beta_{g \mid M^{\prime}}$, a uma seção $S^{\prime}$ de $\beta_{g}$ sobre $M^{\prime} \cup W^{m-1}$. A obstrução à extensão dessa seção a $M^{\prime} \cup W^{m}$ é uma classe em $H^{m}\left(W, M^{\prime} ; \pi_{m-1}(F)\right) \cong H_{1}\left(W, M ; \pi_{m-1}(F)\right)$.

Uma vez que $H_{1}(W ; Z)=0$, temos que $H_{1}\left(W ; \pi_{m-1}(F)\right)=0$; assim, da sequência de homologia do par $(W, M)$, supondo $M$ e $W$ conexas, segue que $H_{1}\left(W, M ; \pi_{m-1}(F)\right)=0$. Portanto, $s^{\prime}$ se estende a uma seção $S$ de $\beta_{g}$ sobre $W$.

Considerando $s=S_{\mid M}$, temos então $l_{f}([s])=\left[M^{\prime}, f^{\prime}, s^{\prime}\right]$. 


\section{Bibliografia}

[1] Brown, E. H. The cohomology of $\mathrm{BSO}_{n}$ and $\mathrm{BO}_{n}$ with integer coefficients. Proc. Amer. Math. Soc., 85, 283-288 (1982).

[ 2 ] Brown, R. L. W. Immersions and embeddings up to cobordism. Can. J. Math. 23, 1102-1115 (1971).

[ 3 ] Conner, P. E. e Floyd, E. E. Differentiable Periodic Maps. Springer Verlag (1979).

[4] Daccach, J. A. Cirurgia e aplicações. Dissertação de Mestrado, ICMSC-USP (1974).

[ 5 ] Dax, J. P. Étude homotopique des espaces de plongements. Ann. Scient. Éc. Norm. Sup. 5, 303-377 (1972).

[6] Gonçalves, D. L. Generalized classes of groups, $\mathcal{C}$-nilpotent spaces and "the Hurewicz Theorem ". Math. Scand., 53, 39-61 (1983).

[ 7 ] Hirsch, M. W. Immersions of manifolds. Trans. Amer. Math. Soc. 93, 242-276 (1959).

[ 8 ] Hu, S-T. Homotopy Theory. Academic Press Inc. (1959).

[ 9 ] Husemoller, D. Fibre bundles. Mc Graw-Hill Book Company (1966).

[10] Koschorke, U. Vector fields and other vector bundle morphisms - A singularity approach. Lecture Notes in Math., 847. Springer Verlag (1981).

[ 11 ] Liao, S. D. On the theory of obstructions of fiber bundles. Ann. of Math. (2) 60, 146191 (1954). 
[ 12 ] Li, B. H. On immersions of manifolds in manifolds. Scientia Sinica Ser. A (25), 255263 (1982).

[ 13 ] On classification of immersions of n-manifolds in (2n-1)-manifolds. Comment. Math. Helv. 57, 135-144 (1982).

[14] Classification of immersions of m-manifolds in $(2 m-1)$ and $(2 m-2)-$ manifolds. Northeastern Math. J., 2 (1), 87-99 (1986).

[15] Immersions of manifolds and stable normal bundles. Sci. Sinica Ser. A $30, n^{\circ} 2,136-147$ (1987).

[ 16 ] Li, B. H. e Peterson, F. P. On immersions of $k$-manifolds in (2k-1)-manifolds. Proc. Amer. Math. Soc. 83, 159-162 (1981).

[17] Immersions of $n$-manifolds into $(2 n-2)$-manifolds. Proc. Amer. Math. Soc. 97, 531-538 (1986).

[ 18 ] Li, G-S. On immersions in bordism classes. Math. Ann. 291, 373-382 (1991).

[ 19 ] . The second normal bordism group of a space pair. Proc. Amer. Math. Soc. $118, \mathrm{n}^{\circ} 1,259-262$ (1993).

[20] Liulevicius, A. Immersions up to cobordism. Ill. J. Math. 19, 149-164 (1973).

[21] Milnor, J. W. A procedure for killing homotopy groups of differentiable manifolds. Differentiable manifolds - Simposia in Pure Mathematics III. AMS (1961).

[22] Milnor, J. W. e Stasheff, J. Characteristic classes. Annals of Mathematics Studies no 76. Princeton Univ. Press (1974). 
[23] Olk, C. Immersionen von mannigfaltigkeiteiten in Euklidische raume. Ph.D Thesis, Siegen Univ. (1980).

[24] Salomonsen, H. A. Bordism and geometric dimension. Math. Scand. 32, 87-111 (1973).

[ 25 ] Spainer, E. H. Algebraic Topology. Mc Graw-Hill Book Company (1966).

[ 26] Steenrod, N. The topology of the fibre bundles. Princeton University Press (1951).

[27 ] Szücs, A. Immersions in bordism classes. Math. Proc.Camb. Philos. Soc. 103, 89-95 (1988).

[ 28 ] Whitehead, G. W. Elements of Homotopy Theory. Graduate Texts in Mathematics, 61. Springer Verlag (1978). 